\title{
MULTIGRID WAVEFORM RELAXATION ON SPATIAL FINITE ELEMENT MESHES: THE DISCRETE-TIME CASE*
}

\author{
JAN JANSSEN $^{\dagger}$ AND STEFAN VANDEWALLE ${ }^{\ddagger}$
}

\begin{abstract}
The efficiency of numerically solving time-dependent partial differential equations on parallel computers can be greatly improved by computing the solution on many time levels simultaneously. The theoretical properties of one such method, namely the discrete-time multigrid waveform relaxation method, are investigated for systems of ordinary differential equations obtained by spatial finite-element discretisation of linear parabolic initial-boundary value problems. The results are compared to the corresponding continuous-time results. The theory is illustrated for a one-dimensional and a two-dimensional model problem and checked against results obtained by numerical experiments.
\end{abstract}

Key words. parabolic partial differential equations, waveform relaxation, multigrid, linear multistep methods

AMS subject classifications. 65F10, 65L05, 65M55, 65M60

1. Introduction. We consider the numerical solution of a linear parabolic initialboundary value problem, spatially discretised by a conforming Galerkin finite-element method. This leads to a linear system of ordinary differential equations (ODEs), see e.g. [10], [20],

$$
B \dot{u}+A u=f, u(0)=u_{0}, t>0,
$$

with $B$ the symmetric positive definite mass matrix, $A$ the stiffness matrix, and $u(t)=$ $\left(u_{1}(t), u_{2}(t), \ldots, u_{d}(t)\right)^{t}$ the unknown solution vector.

In [10] we considered solving (1.1) with the continuous-time multigrid waveform relaxation method. This method is based on waveform relaxation, a highly parallel technique for solving very large systems of ODEs [12], [15]. It is accelerated by using multigrid, a very efficient method for solving elliptic partial differential equations, see e.g. [1], [4], [24]. The continuous-time waveform relaxation method differs from standard ODE solvers in that it computes a solution along a continuous time interval. It requires the analytical solution of certain ODEs and the exact continuous representation of certain functions. The method is therefore mainly of theoretical interest. In an actual implementation of the method, the algorithm is replaced by a discrete-time algorithm. That is, functions are represented discretely as vectors defined on successive time levels, and the ODEs are solved by using standard time-stepping techniques.

The resulting discrete-time multigrid waveform relaxation method belongs to the class of parabolic multigrid methods. These are multigrid methods for time-dependent problems designed to operate on grids extending in space and time. Other examples of such methods are the time-parallel multigrid method [3], [7] and the space-time multigrid method [8]. These methods are highly efficient on parallel computers, possibly outperforming parallel implementations of standard time-stepping methods by orders of magnitude [9], [23]. Their convergence characteristics as iterative solvers are often similar to the convergence characteristics of multigrid methods for stationary problems, although different parabolic multigrid methods may have very different robustness characteristics. The waveform method, in par-

${ }^{*}$ Received by the editors May 20, 1994; accepted for publication (in revised form) March 1, 1995.

${ }^{\dagger}$ Katholieke Universiteit Leuven, Department of Computer Science, Celestijnenlaan 200A, B-3001 Heverlee, Belgium (jan. janssenecs.kuleuven.ac.be). This text presents research results of the Belgian Incentive Program "Information Technology"-Computer Science of the Future, initiated by the Belgian State-Prime Minister's Office for Science, Technology and Culture. The scientific responsibility is assumed by its authors.

$\ddagger$ California Institute of Technology, Applied Mathematics 217-50, Pasadena, CA 91125 (stefan@ama. caltech.edu). This work was supported in part by the NSF under Cooperative Agreement No. CCR-9120008. 
ticular, was shown to be very robust across a wide range of time-discretisation schemes. We refer to [8], [22] for a further discussion.

In this paper, we continue our study of the multigrid waveform relaxation method for systems of the form (1.1). In particular, we analyse the effect of time discretisation when linear multistep formulae are used. The structure of this paper is similar to the structure of [10]. In $\S 2$, we analyse the spectral properties of certain operators that arise in the formulation of the waveform relaxation methods. After a brief review of some definitions and properties of linear multistep methods in $\S 3$, we investigate the convergence of the discrete-time standard waveform relaxation method $(\S 4)$ and of its two-grid acceleration $(\S 5)$, both on finite and infinite time intervals. For systems of the form (1.1) with $B=I$, the discrete-time waveform method and its multigrid variant have been investigated in [14], [16], [17], [21]. Our results are qualitatively very similar, and generalise the ones found in these references. In $\S 6$, we perform a model problem analysis for a one-dimensional and two-dimensional model problem. Finally, in $\S 7$, extensive numerical results are reported.

2. Spectral properties of a special operator. We will show in $\S \S 4$ and 5 that the discretetime waveform relaxation method and its two-grid acceleration can be written as successive approximation schemes of the form

$$
u_{\tau}^{(v)}=\mathcal{H}_{\tau} u_{\tau}^{(\nu-1)}+\varphi_{\tau}
$$

We use subscript $\tau$-notation to denote vectors or sequences, e.g. $u_{\tau}^{(v)}=\left\{u_{i}^{(v)}\right\}_{i=0}^{N-1}$, where $N$ is the (possibly infinite) number of components. Each component is a $d$-vector, and will typically approximate the solution of the system of $d$ differential equations (1.1) at a given time level. Operator $\mathcal{H}_{\tau}$ is a linear discrete convolution operator with matrix-valued kernel $h_{\tau}$,

$$
\left(\mathcal{H}_{\tau} u_{\tau}\right)_{j}=\left(h_{\tau} \star u_{\tau}\right)_{j}=\sum_{i=0}^{j} h_{j-i} u_{i}, j=0, \ldots, N-1
$$

The convergence properties of operator $\mathcal{H}_{\tau}$ will be analysed in the spaces of $\mathbb{C}^{d}$-valued $p$ summable sequences of length $N, l_{p}\left(N ; \mathbb{C}^{d}\right)$, or $l_{p}(N)$ for short. These are Banach spaces with norms given by

$$
\left\|u_{\tau}\right\|_{p}= \begin{cases}\sqrt[p]{\sum_{i=0}^{N-1}\left\|u_{i}\right\|^{p}} & 1 \leq p<\infty \\ \sup _{0 \leq i<N}\left\{\left\|u_{i}\right\|\right\} & p=\infty\end{cases}
$$

with $\|\cdot\|$ any usual $\mathbb{C}^{d}$ vector norm. Recall that the iterative scheme (2.1) is convergent if and only if the spectral radius of $\mathcal{H}_{\tau}$, denoted by $\rho\left(\mathcal{H}_{\tau}\right)$, is smaller than one. The spectral radius is defined as the largest value $\rho$ for which $|\lambda|>\rho$ implies that $\lambda-\mathcal{H}_{\tau}$ has a bounded inverse. When $N$ is finite, it equals the magnitude of the largest eigenvalue of $\mathcal{H}_{\tau}$.

\subsection{Spectral radius on finite time intervals.}

LEMMA 2.1. Consider $\mathcal{H}_{\tau}$ as an operator in $l_{p}(N)$, with $1 \leq p \leq \infty$ and $N$ finite. Then, $\mathcal{H}_{\tau}$ is a bounded operator and

$$
\rho\left(\mathcal{H}_{\tau}\right)=\rho\left(h_{0}\right)=\rho\left(\mathbf{H}_{\tau}(\infty)\right)
$$

with $\mathbf{H}_{\tau}(z)=\sum_{i=0}^{N-1} h_{i} z^{-i}$ the discrete Laplace transform of $h_{\tau}$. 
Proof. Since $\mathcal{H}_{\tau}$ is a linear operator in a finite-dimensional space, boundedness of $\mathcal{H}_{\tau}$ follows. The operation $\mathcal{H}_{\tau} u_{\tau}$ can be represented in standard linear algebra notation as a matrix-vector product,

$$
\left[\begin{array}{cccccc}
h_{0} & & & & & \\
h_{1} & h_{0} & & & & \\
h_{2} & h_{1} & h_{0} & & & \\
\cdot & \cdot & \cdot & \cdot & & \\
\cdot & \cdot & \cdot & \cdot & \cdot & \\
h_{N-1} & \cdot & \cdot & h_{2} & h_{1} & h_{0}
\end{array}\right]\left[\begin{array}{c}
u_{0} \\
u_{1} \\
\cdot \\
\cdot \\
\cdot \\
u_{N-1}
\end{array}\right] .
$$

The spectral radius of operator $\mathcal{H}_{\tau}$ equals the spectral radius of the $N \times N$-block lower triangular Toeplitz matrix in (2.4). By consequence, $\rho\left(\mathcal{H}_{\tau}\right)=\rho\left(h_{0}\right)$. The second equality follows immediately.

\subsection{Spectral radius on infinite time intervals.}

LEMMA 2.2. Suppose $h_{\tau} \in l_{1}(\infty)$, and consider $\mathcal{H}_{\tau}$ as an operator in $l_{p}(\infty)$, with $1 \leq p \leq \infty$. Then, $\mathcal{H}_{\tau}$ is bounded and

$$
\begin{aligned}
\rho\left(\mathcal{H}_{\tau}\right) & =\max _{|z| \geq 1} \rho\left(\mathbf{H}_{\tau}(z)\right) \\
& =\max _{|z|=1} \rho\left(\mathbf{H}_{\tau}(z)\right),
\end{aligned}
$$

with $\mathbf{H}_{\tau}(z)=\sum_{i=0}^{\infty} h_{i} z^{-i}$ the discrete Laplace transform of $h_{\tau}$.

The outline of our proof is very similar to the one given in [16, Thm. 3.1]. Yet, here, it is phrased in terms of general convolution operators. A similar line of arguments is implied in the proof of [14, Prop. 9]. The proof is based on the discrete version of the Paley-Wiener theorem [13]. This theorem states that the solution of a discrete Volterra convolution equation $x_{\tau}+h_{\tau} \star x_{\tau}=f_{\tau}$, with $f_{\tau} \in l_{p}(\infty)$ and $h_{\tau} \in l_{1}(\infty)$ is bounded in $l_{p}(\infty)$ if and only if $\operatorname{det}\left(I+\mathbf{H}_{\tau}(z)\right) \neq 0$ for $|z| \geq 1$, with $\mathbf{H}_{\tau}(z)$ the discrete Laplace transform of $h_{\tau}$.

Proof. The boundedness of $\mathcal{H}_{\tau}$ follows from the fact that $l_{1} \star l_{p} \subset l_{p}$. Indeed, applying Young's inequality for discrete convolution products [6, p. 198] yields

$$
\left\|\mathcal{H}_{\tau} u_{\tau}\right\|_{p} \leq\left\|h_{\tau}\right\|_{1}\left\|u_{\tau}\right\|_{p} .
$$

By definition, the spectral radius of $\mathcal{H}_{\tau}$ is the smallest value of $\rho$ for which $|\lambda|>\rho$ implies that $\lambda-\mathcal{H}_{\tau}$ has a bounded inverse in $l_{p}(\infty)$. Consider

$$
\lambda u_{\tau}-\mathcal{H}_{\tau} u_{\tau}=\lambda u_{\tau}-h_{\tau} \star u_{\tau}=f_{\tau},
$$

with $f_{\tau} \in l_{p}(\infty)$. Suppose $\lambda \neq 0$, then this can be rewritten as a convolution equation

$$
u_{\tau}-\frac{1}{\lambda} h_{\tau} \star u_{\tau}=\frac{1}{\lambda} f_{\tau} .
$$

By the Paley-Wiener theorem, it follows that $u_{\tau}$ is bounded if and only if

$$
\operatorname{det}\left(I-\frac{1}{\lambda} \mathbf{H}_{\tau}(z)\right) \neq 0 \text { for }|z| \geq 1,
$$

or, equivalently,

$$
\rho\left(\mathcal{H}_{\tau}\right)=\sup _{|z| \geq 1} \rho\left(\mathbf{H}_{\tau}(z)\right) .
$$


Note that $\mathbf{H}_{\tau}(z)$ is analytic for $|z|>1$, including $z=\infty$, and, since $h_{\tau} \in l_{1}(\infty)$, it is continuous for $|z| \geq 1$. Also, the spectral radius satisfies the maximum principle. Hence, we obtain (2.5) and (2.6).

Remark 2.1. In the case of $d=1$, this lemma corresponds to a well-known spectral property of semi-infinite Toeplitz operators [18, Thm. 2.1].

In $l_{2}(\infty)$, an analogous result holds for the norm.

LEMMA 2.3. Suppose $h_{\tau} \in l_{1}(\infty)$, and consider $\mathcal{H}_{\tau}$ as an operator in $l_{2}(\infty)$. Denote by $\|\cdot\|_{2}$ the $l_{2}$-norm (2.2) with $\|\cdot\|$ the standard Euclidean vector norm. Then,

$$
\begin{aligned}
\left\|\mathcal{H}_{\tau}\right\|_{2} & =\max _{|z| \geq 1}\left\|\mathbf{H}_{\tau}(z)\right\| \\
& =\max _{|z|=1}\left\|\mathbf{H}_{\tau}(z)\right\|,
\end{aligned}
$$

with $\mathbf{H}_{\tau}(z)$ the discrete Laplace transform of $h_{\tau}$.

Proof. The proof is based on Parseval's relation for vector-valued $l_{2}$-sequences,

$$
\left\|u_{\tau}\right\|_{2}:=\left\|\left\{u_{i}\right\}_{i=0}^{\infty}\right\|_{2}=\left\|\sum_{i=0}^{\infty} u_{i} z^{-i}\right\|_{H_{2}},
$$

where $\|\cdot\|_{H_{2}}$ is the norm in the Hardy-Lebesgue space of square integrable functions analytic outside the unit disk,

$$
\|f(z)\|_{H_{2}}=\sup _{r>1}\left(\frac{1}{2 \pi} \int_{0}^{2 \pi}\left\|f\left(r e^{i \theta}\right)\right\|^{2} d \theta\right)^{1 / 2} .
$$

The Parseval relation for the scalar case can be found, e.g., in [25, p. 41]. By definition of operator norm and by Parseval's relation, we have

$$
\left\|\mathcal{H}_{\tau}\right\|_{2}=\sup \frac{\left\|\mathcal{H}_{\tau} u_{\tau}\right\|_{2}}{\left\|u_{\tau}\right\|_{2}}=\sup \frac{\left\|\mathbf{H}_{\tau}(z) \tilde{u}_{\tau}(z)\right\|_{H_{2}}}{\left\|\tilde{u}_{\tau}(z)\right\|_{H_{2}}},
$$

with $\tilde{u}_{\tau}(z)$ the discrete Laplace transform of $u_{\tau}$. $\left\|\mathcal{H}_{\tau}\right\|_{2}$ can be seen to be equal to $\sup _{|z| \geq 1}\left\|\mathbf{H}_{\tau}(z)\right\|$. (For the technical details of this last step, we refer to the proof of a very similar theorem, [2, Thm. 2.2], which deals with operator norms of Fourier multipliers.) Consideration of the analyticity and continuity of $\mathbf{H}_{\tau}(z)$ leads to (2.7) and (2.8).

Remark 2.2. From (2.3) and (2.5), it follows that the spectral radius of $\mathcal{H}_{\tau}$ on finite time intervals is smaller than the spectral radius of $\mathcal{H}_{\tau}$ on infinite time intervals.

3. Some linear multistep formulae. For the reader's convenience, we recall the general linear multistep formula for calculating the solution to the ODE $\dot{y}=f(t, y)$ with $y(0)=y_{0}$, see e.g. [11, p. 11],

$$
\frac{1}{\tau} \sum_{j=0}^{k} \alpha_{j} y_{n+j}=\sum_{j=0}^{k} \beta_{j} f_{n+j}
$$

In this formula, $\alpha_{j}$ and $\beta_{j}$ are real constants, and $\tau$ denotes a constant step size. We shall assume that $k$ starting values $y_{0}, y_{1}, \ldots, y_{k-1}$ are given. by

DEFINITION 3.1. The characteristic polynomials of the linear multistep method are given

$$
a(z)=\sum_{j=0}^{k} \alpha_{j} z^{j} \quad \text { and } \quad b(z)=\sum_{j=0}^{k} \beta_{j} z^{j}
$$


Throughout this paper we adhere to some common assumptions. The linear multistep method is irreducible: $a(z)$ and $b(z)$ have no common roots; the linear multistep method is consistent: $a(1)=0$ and $a^{\prime}(1)=b(1)$; the linear multistep method is zero-stable: all roots of $a(z)$ are inside the closed unit disk and every root with modulus one is simple. For future reference, we also define the stability region of a linear multistep method, and the related notion of $A(\alpha)$-stability, see e.g. [5], [11].

DEFINITION 3.2. The stability region $S$ consists of those $\mu \in \overline{\mathbb{C}}$ for which the polynomial $a(z)-\mu b(z)$ (around $\mu=\infty: \mu^{-1} a(z)-b(z)$ ) satisfies the root condition: all roots satisfy $\left|z_{j}\right| \leq 1$ and those of modulus one are simple.

DEFINITION 3.3. A multistep method is called

(i) $A(\alpha)$-stable, $0<\alpha<\frac{\pi}{2}$, if $S \supset \Sigma_{\alpha}=\{z:|\operatorname{Arg}(-z)|<\alpha, z \neq 0\}$

(ii) A-stable if $S$ contains the left-half complex plane.

4. The waveform relaxation method. The continuous-time waveform relaxation method for solving initial value problem (1.1) is defined by the splittings $B=M_{B}-N_{B}, A=M_{A}-N_{A}$, and the iteration scheme

$$
M_{B} \dot{u}^{(v)}+M_{A} u^{(v)}=N_{B} \dot{u}^{(v-1)}+N_{A} u^{(\nu-1)}+f,
$$

with $u^{(v)}(0)=u_{0}$. We assume the splitting is such that $M_{B}$ is invertible. This iterative scheme can be written in explicit form as $u^{(v)}=\mathcal{K} u^{(v-1)}+\varphi$. The convergence properties of iteration operator $\mathcal{K}$, the continuous-time waveform relaxation operator, have been studied in [10]. They are expressed in terms of the matrix

$$
\mathbf{K}(z)=\left(z M_{B}+M_{A}\right)^{-1}\left(z N_{B}+N_{A}\right) .
$$

It was shown on finite and infinite time intervals, respectively, and with $i=\sqrt{-1}$, that

$$
\rho(\mathcal{K})=\rho(\mathbf{K}(\infty)) \text { and } \rho(\mathcal{K})=\sup _{\operatorname{Re}(z) \geq 0} \rho(\mathbf{K}(z))=\sup _{\xi \in \mathbb{R}} \rho(\mathbf{K}(i \xi))
$$

4.1. The discrete-time waveform relaxation operator. Application of linear multistep formula (3.1) to the continuous-time iteration scheme (4.1) leads to

$$
\begin{aligned}
& \frac{1}{\tau} \sum_{j=0}^{k} \alpha_{j} M_{B} u_{n+j}^{(\nu)}+\sum_{j=0}^{k} \beta_{j} M_{A} u_{n+j}^{(v)} \\
& =\frac{1}{\tau} \sum_{j=0}^{k} \alpha_{j} N_{B} u_{n+j}^{(\nu-1)}+\sum_{j=0}^{k} \beta_{j} N_{A} u_{n+j}^{(\nu-1)}+\sum_{j=0}^{k} \beta_{j} f_{n+j}, n \geq 0 .
\end{aligned}
$$

We do not iterate on the $k$ starting values, i.e., $u_{j}^{(v)}=u_{j}^{(v-1)}=u_{j}$, for $j<k$. In the remainder of the text we shall concentrate on the use of implicit methods, i.e., $\beta_{k} \neq 0$. Equation (4.4) can then be solved uniquely for every $n$ if and only if the following condition is satisfied:

$$
\frac{\alpha_{k}}{\beta_{k}} \notin \sigma\left(-\tau M_{B}^{-1} M_{A}\right),
$$

where $\sigma(\cdot)$ denotes the spectrum. Further on we shall refer to this condition as the discrete solvability condition.

Iteration (4.4) can be rewritten as $u_{\tau}^{(\nu)}=\mathcal{K}_{\tau} u_{\tau}^{(\nu-1)}+\varphi_{\tau}$. Because we do not iterate on the starting values, we use a slightly different subscript $\tau$-notation here than the one in (2.1), that is,

$$
u_{\tau}=\left\{u_{k+i}\right\}_{i=0}^{N-1}
$$


(Alternatively, we could have used negative indices to denote the time levels associated with the $k$ starting values, as is done in [13], [14]. This, however, would require some shifting in the indices of formulae (3.1) and (4.4).) The precise expression for $\varphi_{\tau}$ can be calculated following the lines of [17, p. 536-537]. It depends on the values of $f_{n}, n \geq 0$ and on the starting values $u_{n}, n<k$. In order to determine the nature of $\mathcal{K}_{\tau}$, the discrete-time waveform relaxation operator, we rewrite (4.4) using $e_{n}^{(v)}=u_{n}^{(v)}-u_{n}$. Here, $u_{n}$ is the exact solution of ODE (1.1) when discretised using the linear multistep method. This gives

$$
\frac{1}{\tau} \sum_{j=0}^{k} \alpha_{j} M_{B} e_{n+j}^{(\nu)}+\sum_{j=0}^{k} \beta_{j} M_{A} e_{n+j}^{(\nu)}=\frac{1}{\tau} \sum_{j=0}^{k} \alpha_{j} N_{B} e_{n+j}^{(\nu-1)}+\sum_{j=0}^{k} \beta_{j} N_{A} e_{n+j}^{(\nu-1)}, \quad n \geq 0 .
$$

With $C_{j}=\frac{1}{\tau} \alpha_{j} M_{B}+\beta_{j} M_{A}$, and $D_{j}=\frac{1}{\tau} \alpha_{j} N_{B}+\beta_{j} N_{A}$, this becomes

$$
\sum_{j=0}^{k} C_{j} e_{n+j}^{(\nu)}=\sum_{j=0}^{k} D_{j} e_{n+j}^{(\nu-1)}, n \geq 0 .
$$

Note that $e_{j}^{(\nu)}=e_{j}^{(\nu-1)}=0, j<k$. When we combine the first $N$ equations, i.e., the equations for the unknowns on time steps $k, \ldots, N+k-1$, and after introducing vector $E^{(\nu)}=\left[e_{k}^{(v)} e_{k+1}^{(\nu)} \ldots e_{N+k-1}^{(\nu)}\right]^{t}$, we get

$$
E^{(\nu)}=C^{-1} D E^{(\nu-1)} .
$$

Matrices $C$ and $D$ are $N \times N$-block lower triangular matrices with $k+1$ constant diagonals. The blocks on the $j$ th diagonal are given respectively by $C_{k-j}$ and $D_{k-j}$. It follows immediately that matrix $C^{-1} D$ is a $N \times N$-block lower triangular Toeplitz matrix. Hence, $\mathcal{K}_{\tau}$ is a discrete linear convolution operator on the $l_{p}$-space of vectors or sequences of length $N$. The $j$ th component of the matrix-valued discrete convolution kernel $k_{\tau}$ equals the (constant) submatrix on the $j$ th lower block diagonal of $C^{-1} D$.

In the theory we shall need the discrete Laplace transform of the convolution kernel. It can be found by discrete Laplace-transforming equation (4.7). If $\tilde{e}_{\tau}^{(\nu)}(z)$ denotes the transform of $e_{\tau}^{(v)}$, we obtain

$$
\tilde{e}_{\tau}^{(v)}(z)=\mathbf{K}_{\tau}(z) \tilde{e}_{\tau}^{(\nu-1)}(z),
$$

with the discrete-time waveform relaxation matrix given by

$$
\mathbf{K}_{\tau}(z)=\left(a(z) M_{B}+\tau b(z) M_{A}\right)^{-1}\left(a(z) N_{B}+\tau b(z) N_{A}\right) .
$$

By comparison to (4.2) the following relation results:

$$
\mathbf{K}_{\tau}(z)=\mathbf{K}\left(\frac{1}{\tau} \frac{a}{b}(z)\right) .
$$

Note that (4.10) still holds when $\frac{a}{b}(z)$ is set to $\infty$ in the case of $b(z)=0$. (In this case $a(z) \neq 0$, since the characteristic polynomials have no common roots.)

\subsection{Convergence analysis.}

4.2.1. Convergence on finite time intervals.

THEOREM 4.1. Assume that condition (4.5) is satisfied, and consider $\mathcal{K}_{\tau}$ as an operator in $l_{p}(N)$, with $1 \leq p \leq \infty$ and $N$ finite. Then, $\mathcal{K}_{\tau}$ is bounded and

$$
\rho\left(\mathcal{K}_{\tau}\right)=\rho\left(\mathbf{K}\left(\frac{1}{\tau} \frac{\alpha_{k}}{\beta_{k}}\right)\right) .
$$


Proof. The theorem follows from Lemma 2.1 and the observation that

$$
\lim _{z \rightarrow \infty} \mathbf{K}_{\tau}(z)=\lim _{z \rightarrow \infty} \mathbf{K}\left(\frac{1}{\tau} \frac{a}{b}(z)\right)=\mathbf{K}\left(\frac{1}{\tau} \frac{\alpha_{k}}{\beta_{k}}\right) .
$$

4.2.2. Convergence on infinite time intervals. The following lemma deals with the boundedness of the discrete-time waveform relaxation operator $\mathcal{K}_{\tau}$. It is proved using a matrix-valued version of Wiener's inversion theorem [13, p. 446] and [16, p. 577], which is stated here for the reader's convenience.

THEOREM 4.2 (WIENER'S INVERSION THEOREM). Given a matrix-valued sequence $A_{\tau}$ such that $A_{\tau} \in l_{1}(\infty)$, assume that

$$
\operatorname{det} \sum_{i=0}^{\infty} A_{i} z^{-i} \neq 0
$$

for $|z| \geq 1$. Setting $\sum_{i=0}^{\infty} B_{i} z^{-i}=\left(\sum_{i=0}^{\infty} A_{i} z^{-i}\right)^{-1}$, we have $B_{\tau} \in l_{1}(\infty)$.

LEMMA 4.3. If $\sigma\left(-\tau M_{B}^{-1} M_{A}\right) \subset$ int $S$, then $\mathcal{K}_{\tau}$ is bounded in $l_{p}(\infty)$.

Proof. It is sufficient to prove that the kernel $k_{\tau}$ of the discrete convolution operator $\mathcal{K}_{\tau}$ is an $l_{1}$-sequence. To this end, consider first the $l_{1}$-sequence

$$
\alpha_{k} M_{B}+\tau \beta_{k} M_{A}, \alpha_{k-1} M_{B}+\tau \beta_{k-1} M_{A}, \ldots, \alpha_{0} M_{B}+\tau \beta_{0} M_{A}, 0,0, \ldots
$$

Its discrete Laplace transform equals the matrix function $z^{-k}\left(a(z) M_{B}+\tau b(z) M_{A}\right)$. By Wiener's theorem, we have that the inverse, $\left(a(z) M_{B}+\tau b(z) M_{A}\right)^{-1} z^{k}$, is the transform of another $l_{1}$-sequence, say $r_{\tau}$, if

$$
\operatorname{det}\left(a(z) M_{B}+\tau b(z) M_{A}\right) \neq 0 \text { for }|z| \geq 1 .
$$

Next, consider the $l_{1}$-sequence

$$
s_{\tau}=\alpha_{k} N_{B}+\tau \beta_{k} N_{A}, \alpha_{k-1} N_{B}+\tau \beta_{k-1} N_{A}, \ldots, \alpha_{0} N_{B}+\tau \beta_{0} N_{A}, 0,0, \ldots,
$$

the discrete Laplace transform of which is given by $z^{-k}\left(a(z) N_{B}+\tau b(z) N_{A}\right)$. The convolution of $r_{\tau}$ and $s_{\tau}$ is another $l_{1}$-sequence, which can be seen to be equal to the kernel $k_{\tau}$. Indeed, the discrete Laplace transform of $r_{\tau} \star s_{\tau}$ is identical to $\mathbf{K}_{\tau}(z)$. As a result, it follows that $\mathcal{K}_{\tau}$ is bounded if (4.12) is satisfied.

Suppose there is a $z$ with $|z| \geq 1$ such that

$$
\operatorname{det}\left(a(z) M_{B}+\tau b(z) M_{A}\right)=0 .
$$

Then necessarily $b(z) \neq 0$. (If $b(z)=0$ then $a(z) \neq 0$, because $a(z)$ and $b(z)$ have no common roots. Since $M_{B}$ is assumed to be invertible, equality (4.13) cannot hold.) Hence, we obtain

$$
\operatorname{det}\left(\frac{a}{b}(z) M_{B}+\tau M_{A}\right)=0,
$$

and therefore $\frac{a}{b}(z) \in \sigma\left(-\tau M_{B}^{-1} M_{A}\right)$. Since $|z| \geq 1$, it follows that $-\tau M_{B}^{-1} M_{A}$ has an eigenvalue which is not an interior point of $S$. This contradicts the assumption of the lemma. Hence, (4.12) is satisfied.

Remark 4.1. Condition $\sigma\left(-\tau M_{B}^{-1} M_{A}\right) \subset$ int $S$ implies the discrete solvability condition (4.5). Indeed, since $\frac{\alpha_{k}}{\beta_{k}}=\frac{a}{b}(\infty)$, it follows that $\frac{\alpha_{k}}{\beta_{k}} \notin$ int $S$, and, therefore, $\frac{\alpha_{k}}{\beta_{k}} \notin \sigma\left(-\tau M_{B}^{-1} M_{A}\right)$. 
Remark 4.2. Condition $\sigma\left(-\tau M_{B}^{-1} M_{A}\right) \subset$ int $S$ implies that all poles of $\mathbf{K}(z)$ are in the interior of the scaled stability region $\frac{1}{\tau} S$.

THEOREM 4.4. Assume $\sigma\left(-\tau M_{B}^{-1} M_{A}\right) \subset$ int $S$, and consider $\mathcal{K}_{\tau}$ as an operator in $l_{p}(\infty)$, with $1 \leq p \leq \infty$. Then,

$$
\begin{aligned}
\rho\left(\mathcal{K}_{\tau}\right) & =\sup \{\rho(\mathbf{K}(z)) \mid \tau z \in \mathbb{C} \backslash \text { int } S\} \\
& =\sup _{\tau z \in \partial S} \rho(\mathbf{K}(z))
\end{aligned}
$$

Proof. As $\sigma\left(-\tau M_{B}^{-1} M_{A}\right) \subset$ int $S$, it follows that $k_{\tau} \in l_{1}(\infty)$. Lemma 2.2 yields

$$
\rho\left(\mathcal{K}_{\tau}\right)=\max _{|z| \geq 1} \rho\left(\mathbf{K}_{\tau}(z)\right)=\max _{|z| \geq 1} \rho\left(\mathbf{K}\left(\frac{1}{\tau} \frac{a}{b}(z)\right)\right) .
$$

By definition of the stability region,

$$
\overline{\mathbb{C}} \backslash \text { int } S=\left\{\frac{a}{b}(z):|z| \geq 1\right\},
$$

and thereby (4.14) follows. Equality (4.15) is obtained by the maximum principle. Note that we write "sup" instead of "max," since the maximum may be approached at infinity.

In $l_{2}(\infty)$, a similar result holds for the norm by application of Lemma 2.3.

THEOREM 4.5. Assume $\sigma\left(-\tau M_{B}^{-1} M_{A}\right) \subset$ int $S$, and consider $\mathcal{K}_{\tau}$ as an operator in $l_{2}(\infty)$. Denote by $\|\cdot\|_{2}$ the $l_{2}$-norm (2.2) with $\|\cdot\|$ the standard Euclidean vector norm. Then,

$$
\begin{aligned}
\left\|\mathcal{K}_{\tau}\right\|_{2} & =\sup \{\|\mathbf{K}(z)\|: \tau z \in \mathbb{C} \backslash \text { int } S\} \\
& =\sup _{\tau z \in \partial S}\|\mathbf{K}(z)\| .
\end{aligned}
$$

Analogous to the discussion in [17, Thm. 4.2], we can make the following note.

Remark 4.3. When the assumption in the above theorems is violated, a weaker condition may be satisfied: $\sigma\left(-\tau M_{B}^{-1} M_{A}\right) \subset$ int $S_{\gamma \tau}$, where $S_{\gamma \tau}$ consists of all $\mu$ for which $a\left(e^{-\gamma \tau} z\right)-$ $\mu b\left(e^{-\gamma \tau} z\right.$ ) (around $\mu=\infty: \mu^{-1} a\left(e^{-\gamma \tau} z\right)-b\left(e^{-\gamma \tau} z\right)$ ) satisfies the root condition. The analysis then can be redone using an exponentially scaled norm,

$$
\left\|u_{\tau}\right\|_{\gamma}=\left\|\left\{u_{i}\right\}\right\|_{\gamma}=\left\|\left\{e^{-\gamma \tau i} u_{i}\right\}\right\| .
$$

The norm in the right-hand side is a standard $p$-norm (2.2). With this change of norm, the suprema in Theorems 4.4 and 4.5 have to be taken over all $\tau z$ in $\mathbb{C} \backslash$ int $S_{\gamma \tau}$, or, after application of the maximum principle, over $\partial S_{\gamma \tau}$.

4.3. Discrete-time versus continuous-time results. The continuous-time results (4.3) are regained when we let $\tau \rightarrow 0$ in the convergence formulae for operator $\mathcal{K}_{\tau}$. For finite time intervals, we have

$$
\lim _{\tau \rightarrow 0} \rho\left(\mathcal{K}_{\tau}\right)=\lim _{\tau \rightarrow 0} \rho\left(\mathbf{K}\left(\frac{1}{\tau} \frac{\alpha_{k}}{\beta_{k}}\right)\right)=\rho(\mathbf{K}(\infty))=\rho(\mathcal{K}) .
$$

A similar result is found for infinite time intervals. Note that the tangent to $\partial S$ in the origin of the complex plane is the imaginary axis, for any consistent linear multistep method. As such, the boundary of the scaled stability region $\partial\left(\frac{1}{\tau} S\right)$ tends to the imaginary axis when $\tau \rightarrow 0$. Consequently,

$$
\lim _{\tau \rightarrow 0} \rho\left(\mathcal{K}_{\tau}\right)=\lim _{\tau \rightarrow 0} \sup _{\tau z \in \partial S} \rho(\mathbf{K}(z))=\sup _{\xi \in \mathbb{R}} \rho(\mathbf{K}(i \xi))=\rho(\mathcal{K}) .
$$


Furthermore, for a fixed time step $\tau$, we can prove the following theorem for $A(\alpha)$-stable linear multistep methods (see Definition 3.3). The theorem is closely related to [14, Prop. 9], where multigrid waveform relaxation on finite-difference grids is analysed. We reformulate the proof, using our notations, for completeness.

THEOREM 4.6. Assume $\sigma\left(-\tau M_{B}^{-1} M_{A}\right) \subset \Sigma_{\alpha}$. Consider $\mathcal{K}_{\tau}$ as an operator in $l_{p}(\infty)$ and $\mathcal{K}$ as an operator in $L_{p}(0, \infty)$, with $1 \leq p \leq \infty$. Then,

(i) if the linear multistep method is A-stable, then $\rho\left(\mathcal{K}_{\tau}\right) \leq \rho(\mathcal{K})$;

(ii) if the linear multistep method is $A(\alpha)$-stable, then

$$
\rho\left(\mathcal{K}_{\tau}\right) \leq \sup _{z \in \Sigma_{\alpha}^{c}} \rho(\mathbf{K}(z))=\sup _{z \in \partial \Sigma_{\alpha}^{c}} \rho(\mathbf{K}(z)),
$$

with $\Sigma_{\alpha}^{c}=\mathbb{C} \backslash \Sigma_{\alpha}=\{z:|\operatorname{Arg}(z)| \leq \pi-\alpha\}$.

Proof. Part (i) is a special case of (ii) with $\alpha=\pi / 2$, combined with the second equality of (4.3). For part (ii), we notice that we may apply Theorem 4.4 since $\sigma\left(-\tau M_{B}^{-1} M_{A}\right) \subset \Sigma_{\alpha} \subset$ int $S$. Therefore,

$$
\rho\left(\mathcal{K}_{\tau}\right)=\max _{|z| \geq 1} \rho\left(\mathbf{K}\left(\frac{1}{\tau} \frac{a}{b}(z)\right)\right) .
$$

If the multistep method is $A(\alpha)$-stable, then $\frac{a}{b}(z) \in \Sigma_{\alpha}^{c}$ for $|z| \geq 1$. Combining the latter with (4.20) yields the inequality of (4.19). The equality is obtained by the maximum principle.

5. The multigrid waveform relaxation method. The splittings of matrices $B$ and $A$ used in actual computations typically correspond to Gauss-Seidel or weighted Jacobi splittings. Each iteration defined by (4.1) can then be computed as the solution of $d$ ordinary differential equations, each in a single unknown. The resulting iteration can be accelerated by using the multigrid principle, in a very similar way as the standard pointwise relaxation methods are accelerated when solving elliptic partial differential equations.

The continuous-time two-grid waveform relaxation scheme is sketched below. We refer to [10] for a more elaborate description. The algorithm uses two nested grids, a coarse grid $\Omega_{H}$ and a fine grid $\Omega_{h}$. Grid functions are mapped from the one grid to the other by a prolongation (or interpolation) operator $\left(p: \Omega_{H} \rightarrow \Omega_{h}\right)$ and a restriction operator $\left(r: \Omega_{h} \rightarrow \Omega_{H}\right)$. The discretisation on the fine grid is defined by the matrices $B_{h}$ and $A_{h}$, the discretisation on the coarse grid by $B_{H}$ and $A_{H}$. One iteration transforms iterate $u^{(v-1)}$ into $u^{(v)}$ in three steps.

(i) Presmoothing. Set $x_{h}^{(0)}=u^{(\nu-1)}$ and perform $v_{1}$ fine-grid waveform relaxation steps: for $v=1,2, \ldots, v_{1}$, solve

$$
M_{B_{h}} \dot{x}_{h}^{(\nu)}+M_{A_{h}} x_{h}^{(\nu)}=N_{B_{h}} \dot{x}_{h}^{(\nu-1)}+N_{A_{h}} x_{h}^{(\nu-1)}+f_{h}, \text { with } x_{h}^{(\nu)}(0)=u_{0} .
$$

(ii) Coarse-grid correction. Calculate the defect

$$
d_{h}=B \dot{x}_{h}^{\left(\nu_{1}\right)}+A_{h} x_{h}^{\left(\nu_{1}\right)}-f_{h} .
$$

Solve the coarse-grid defect equation

$$
B_{H} \dot{v}_{H}+A_{H} v_{H}=r d_{h} \text {, with } v_{H}(0)=0,
$$

and correct,

$$
\bar{x}_{h}=x_{h}^{\left(\nu_{1}\right)}-p v_{H} .
$$

(iii) Postsmoothing. Set $x_{h}^{(0)}=\bar{x}_{h}$ and perform $v_{2}$ fine-grid waveform relaxation steps (5.1). Set $u^{(v)}=x_{h}^{\left(\nu_{2}\right)}$. 
This two-grid cycle can be written as $u^{(\nu)}=\mathcal{M} u^{(\nu-1)}+\varphi$, where $\mathcal{M}$ is called the continuous-time two-grid waveform relaxation operator. The convergence formulae of $\mathcal{M}$ as an iteration operator resemble those of the standard waveform relaxation method. More precisely, in [10] we find for the finite and for the infinite time-interval case, respectively,

$$
\rho(\mathcal{M})=\rho(\mathbf{M}(\infty)) \quad \text { and } \quad \rho(\mathcal{M})=\sup _{\operatorname{Re}(z) \geq 0} \rho(\mathbf{M}(z))=\sup _{\xi \in \mathbb{R}} \rho(\mathbf{M}(i \xi)) .
$$

$\mathbf{M}(z)$, the continuous-time two-grid waveform relaxation matrix, is given by

$$
\mathbf{M}(z)=\mathbf{K}^{\nu_{2}}(z)\left(I-p\left(z B_{H}+A_{H}\right)^{-1} r\left(z B_{h}+A_{h}\right)\right) \mathbf{K}^{\nu_{1}}(z),
$$

with $\mathbf{K}(z)$ the fine-grid matrix given in (4.2). We recall from [10, Rem. 4.1] the following important remark.

Remark 5.1. In the case of a Gauss-Seidel (or weighted Jacobi) splitting of $A_{h}$ and $B_{h}$, $\mathbf{K}(z)$ and $\mathbf{M}(z)$ are respectively the Gauss-Seidel (or weighted Jacobi) iteration matrix and the two-grid iteration matrix for the system $\left(z B_{h}+A_{h}\right) u_{h}=f_{h}$.

In the following, the discrete-time variant of this two-grid waveform relaxation method is theoretically investigated. We refer to the Appendix for a similar convergence study of the multigrid waveform relaxation method. The latter is defined by solving the coarse-grid defect equation using one or more similar two-grid waveform relaxation cycles, and applying this idea recursively.

5.1. The discrete-time two-grid waveform relaxation operator. We discretise the equations of the continuous-time two-grid cycle using a linear multistep method with a fixed time step $\tau$. As before, we assume that we do not iterate on the $k$ given starting values. The discrete-time two-grid cycle defines a linear operator $\mathcal{M}_{\tau}$, which satisfies

$$
u_{\tau}^{(v)}=\mathcal{M}_{\tau} u_{\tau}^{(\nu-1)}+\varphi_{\tau} \text { and } e_{\tau}^{(\nu)}=\mathcal{M}_{\tau} e_{\tau}^{(\nu-1)},
$$

where $e_{\tau}^{(\nu)}$ is the error of the $\nu$ th iterate. Our notation is again similar to (4.6). $\mathcal{M}_{\tau}$ is called the discrete-time two-grid waveform relaxation operator.

The second equation of (5.3) can be reformulated similar to how we arrived at (4.8),

$$
E^{(v)}=\left(C_{h}^{-1} D_{h}\right)^{\nu_{2}}\left(I-P F_{H}^{-1} R F_{h}\right)\left(C_{h}^{-1} D_{h}\right)^{\nu_{1}} E^{(\nu-1)} .
$$

Here, $E^{(\nu)}=\left[e_{k}^{(\nu)} e_{k+1}^{(\nu)} \ldots e_{N+k-1}^{(\nu)}\right]^{t}$. Matrices $C_{h}, D_{h}, F_{H}$ and $F_{h}$ are $N \times N$-block lower triangular matrices with $k+1$ constant diagonals. The blocks of the $j$ th diagonal equal $\left(C_{h}\right)_{k-j},\left(D_{h}\right)_{k-j},\left(F_{H}\right)_{k-j}$, and $\left(F_{h}\right)_{k-j}$, respectively, with

$$
\left(C_{h}\right)_{j}=\frac{1}{\tau} \alpha_{j} M_{B_{h}}+\beta_{j} M_{A_{h}},\left(D_{h}\right)_{j}=\frac{1}{\tau} \alpha_{j} N_{B_{h}}+\beta_{j} N_{A_{h}}
$$

and

$$
\left(F_{H}\right)_{j}=\frac{1}{\tau} \alpha_{j} B_{H}+\beta_{j} A_{H}, \quad\left(F_{h}\right)_{j}=\frac{1}{\tau} \alpha_{j} B_{h}+\beta_{j} A_{h} .
$$

Matrices $P$ and $R$ are block diagonal with constant diagonal blocks equal to matrices $p$ and $r$, respectively. $I$ is the identity matrix of dimension $d \times N$. The resulting discrete-time two-grid cycle is well defined, if and only if the following conditions hold:

$$
\frac{\alpha_{k}}{\beta_{k}} \notin \sigma\left(-\tau M_{B_{h}}^{-1} M_{A_{h}}\right) \text { and } \frac{\alpha_{k}}{\beta_{k}} \notin \sigma\left(-\tau B_{H}^{-1} A_{H}\right) .
$$

We shall refer to (5.5) as the discrete solvability conditions for the two-grid algorithm. 
It can be seen that the matrix premultiplying $E^{(v-1)}$ in (5.4) is a lower triangular block Toeplitz matrix. This implies that $\mathcal{M}_{\tau}$ is a discrete linear convolution operator. The discrete Laplace transform of its matrix-valued kernel can be found by transforming the equations of the discrete-time two-grid cycle. It is denoted by $\mathbf{M}_{\tau}(z)$, the discrete-time two-grid waveform relaxation matrix, and equals

$$
\mathbf{M}_{\tau}(z)=\mathbf{K}_{\tau}{ }^{\nu_{2}}(z) \mathbf{C}_{\tau}(z) \mathbf{K}_{\tau}^{\nu_{1}}(z)
$$

with $\mathbf{K}_{\tau}(z)$ given by (4.9) and $\mathbf{C}_{\tau}(z)$ given by

$$
I-p\left(a(z) B_{H}+\tau b(z) A_{H}\right)^{-1} r\left(a(z) B_{h}+\tau b(z) A_{h}\right) .
$$

Matrix $\mathbf{M}_{\tau}(z)$ satisfies a similar relation as $\mathbf{K}_{\tau}(z)$ does in (4.10):

$$
\mathbf{M}_{\tau}(z)=\mathbf{M}\left(\frac{1}{\tau} \frac{a}{b}(z)\right) .
$$

5.2. Convergence analysis. The convergence analysis of operator $\mathcal{M}_{\tau}$ is very similar to the convergence analysis of the standard waveform relaxation operator $\mathcal{K}_{\tau}$.

\subsubsection{Convergence on finite time intervals.}

THEOREM 5.1. Assume that conditions (5.5) are satisfied, and consider $\mathcal{M}_{\tau}$ as an operator in $l_{p}(N)$, with $1 \leq p \leq \infty$ and $N$ finite. Then, $\mathcal{M}_{\tau}$ is bounded and

$$
\rho\left(\mathcal{M}_{\tau}\right)=\rho\left(\mathbf{M}\left(\frac{1}{\tau} \frac{\alpha_{k}}{\beta_{k}}\right)\right) \text {. }
$$

Proof. The theorem follows from Lemma 2.1 and (5.6):

$$
\rho\left(\mathcal{M}_{\tau}\right)=\rho\left(\mathbf{M}_{\tau}(\infty)\right)=\rho\left(\mathbf{M}\left(\frac{1}{\tau} \frac{a}{b}(\infty)\right)\right)=\rho\left(\mathbf{M}\left(\frac{1}{\tau} \frac{\alpha_{k}}{\beta_{k}}\right)\right) .
$$

5.2.2. Convergence on infinite time intervals. We first prove the boundedness of $\mathcal{M}_{\tau}$, i.e., we prove the two-grid equivalent of Lemma 4.3.

LEMMA 5.2. Assume $\sigma\left(-\tau M_{B_{h}}^{-1} M_{A_{h}}\right) \cup \sigma\left(-\tau B_{H}^{-1} A_{H}\right) \subset$ int $S$. Then, $\mathcal{M}_{\tau}$ is bounded in $l_{p}(\infty)$.

Proof. It is sufficient to prove that the kernel of $\mathcal{M}_{\tau}$ belongs to $l_{1}(\infty)$. We shall analyse each of the factors in the formula for $\mathbf{M}_{\tau}(z)$ separately.

We have, from the proof of Lemma 4.3, that $\mathbf{K}_{\tau}(z)$ is the discrete Laplace transform of an $l_{1}$-sequence, say $q_{\tau}$, if

$$
\operatorname{det}\left(a(z) M_{B_{h}}+\tau b(z) M_{A_{h}}\right) \neq 0,|z| \geq 1 .
$$

Consider the $l_{1}$-sequence

$$
\alpha_{k} B_{H}+\tau \beta_{k} A_{H}, \alpha_{k-1} B_{H}+\tau \beta_{k-1} A_{H}, \ldots, \alpha_{0} B_{H}+\tau \beta_{0} A_{H}, 0,0, \ldots
$$

Its transform is given by $z^{-k}\left(a(z) B_{H}+\tau b(z) A_{H}\right)$. By Wiener's inversion theorem, $\left(a(z) B_{H}+\right.$ $\left.\tau b(z) A_{H}\right)^{-1} z^{k}$ is the transform of an $l_{1}$-sequence, say $w_{\tau}$, if

$$
\operatorname{det}\left(a(z) B_{H}+\tau b(z) A_{H}\right) \neq 0,|z| \geq 1 .
$$

Next, consider the $l_{1}$-sequences

$$
\begin{aligned}
i_{\tau} & =I, 0, \ldots, 0,0,0, \ldots, \\
v_{\tau} & =\alpha_{k} B_{h}+\tau \beta_{k} A_{h}, \alpha_{k-1} B_{h}+\tau \beta_{k-1} A_{h}, \ldots, \alpha_{0} B_{h}+\tau \beta_{0} A_{h}, 0,0, \ldots
\end{aligned}
$$


$I$ is the $d \times d$ identity matrix. Their transforms are given, respectively, by

$$
I \quad \text { and } \quad z^{-k}\left(a(z) B_{h}+\tau b(z) A_{h}\right) .
$$

Now, consider the sequence

$$
\underbrace{q_{\tau} \star q_{\tau} \star \cdots \star q_{\tau}}_{\nu_{2} \text { times }} \star\left(i_{\tau}-p w_{\tau} \star r v_{\tau}\right) \star \underbrace{q_{\tau} \star q_{\tau} \star \cdots \star q_{\tau}}_{\nu_{1} \text { times }} .
$$

If conditions (5.8) and (5.9) are satisfied, it follows that this sequence is in $l_{1} .\left(l_{1}\right.$ is closed under convolution and addition. The multiplication of an $l_{1}$-sequence by a matrix is an $l_{1}$-sequence.) The discrete Laplace transform of sequence (5.10) equals $\mathbf{M}_{\tau}(z)$, hence the sequence equals the kernel of $\mathcal{M}_{\tau}$. To conclude, $\mathcal{M}_{\tau}$ is bounded under conditions (5.8) and (5.9).

Suppose one of these conditions is violated. That is to say, there is a $z$ with $|z| \geq 1$ such that $\operatorname{det}\left(a(z) M_{B_{h}}+\tau b(z) N_{B_{h}}\right)=0$ or $\operatorname{det}\left(a(z) B_{H}+\tau b(z) A_{H}\right)=0$. That would mean that $\frac{a}{b}(z) \in \sigma\left(-\tau M_{B_{h}}^{-1} M_{A_{h}}\right) \cup \sigma\left(-\tau B_{H}^{-1} A_{H}\right)$. Since $|z| \geq 1$ this violates the assumption of the lemma.

Remark 5.2. The assumption of Lemma 5.2 implies the two-grid discrete solvability conditions (5.5).

Remark 5.3. The assumption of Lemma 5.2 implies that all poles of $\mathbf{M}(z)$ are inside the scaled stability region $\frac{1}{\tau} S$.

THEOREM 5.3. Assume $\sigma\left(-\tau M_{B_{h}}^{-1} M_{A_{h}}\right) \cup \sigma\left(-\tau B_{H}^{-1} A_{H}\right) \subset$ int $S$, and consider $\mathcal{M}_{\tau}$ as an operator in $l_{p}(\infty)$, with $1 \leq p \leq \infty$. Then,

$$
\begin{aligned}
\rho\left(\mathcal{M}_{\tau}\right) & =\sup \{\rho(\mathbf{M}(z)) \mid \tau z \in \mathbb{C} \backslash \text { int } S\} \\
& =\sup _{\tau z \in \partial S} \rho(\mathbf{M}(z)) .
\end{aligned}
$$

Proof. The proof is a direct consequence of Lemma 2.2, and is similar to the proof of Theorem 4.4.

Application of Lemma 2.3 yields the following result for the $l_{2}$-norm of $\mathcal{M}_{\tau}$.

THEOREM 5.4. Assume $\sigma\left(-\tau M_{B_{h}}^{-1} M_{A_{h}}\right) \cup \sigma\left(-\tau B_{H}^{-1} A_{H}\right) \subset$ int $S$, and consider $\mathcal{M}_{\tau}$ as an operator in $l_{2}(\infty)$. Denote by $\|\cdot\|_{2}$ the $l_{2}$-norm (2.2) with $\|\cdot\|$ the standard Euclidean vector norm. Then,

$$
\begin{aligned}
\left\|\mathcal{M}_{\tau}\right\|_{2} & \left.=\sup _{\{}\|\mathbf{M}(z)\|: \tau z \in \mathbb{C} \backslash \text { int } S\right\} \\
& =\sup _{\tau z \in \partial S}\|\mathbf{M}(z)\| .
\end{aligned}
$$

Remark 5.4. If the assumption of the former theorems is violated, but the weaker condition $\sigma\left(-\tau M_{B_{h}}^{-1} M_{A_{h}}\right) \cup \sigma\left(-\tau B_{H}^{-1} A_{H}\right) \subset$ int $S_{\gamma \tau}$ holds, then we can formulate a remark analogous to Remark 4.3.

5.3. Discrete-time versus continuous-time results. The relation between the two-grid operators $\mathcal{M}_{\tau}$ and $\mathcal{M}$ is similar to the relation between $\mathcal{K}_{\tau}$ and $\mathcal{K}$. More precisely, for both finite and infinite intervals,

$$
\lim _{\tau \rightarrow 0} \rho\left(\mathcal{M}_{\tau}\right)=\rho(\mathcal{M})
$$

We also state the two-grid equivalent of Theorem 4.6, without proof.

THEOREM 5.5. Assume $\sigma\left(-\tau M_{B_{h}}^{-1} M_{A_{h}}\right) \cup \sigma\left(-\tau B_{H}^{-1} A_{H}\right) \subset \Sigma_{\alpha}$. Consider $\mathcal{M}_{\tau}$ as an operator in $l_{p}(\infty)$ and $\mathcal{M}$ as an operator in $L_{p}(0, \infty)$, with $1 \leq p \leq \infty$. Then, 
TABLE 6.1

Theoretical and measured values of $\rho\left(\mathcal{K}_{\tau}\right)$ for $(6.1)(h=1 / 16, \tau=1 / 100)$.

\begin{tabular}{ccccccc}
\hline & $\mathrm{CN}$ & $\mathrm{BDF}(1)$ & $\mathrm{BDF}(2)$ & $\mathrm{BDF}(3)$ & $\mathrm{BDF}(4)$ & $\mathrm{BDF}(5)$ \\
\hline \hline finite length & 0.458 & 0.658 & 0.548 & 0.486 & 0.445 & 0.414 \\
\hline infinite length & 0.962 & 0.962 & 0.962 & 0.976 & 1.149 & 1.865 \\
\hline measured & 0.960 & 0.961 & 0.961 & 0.974 & 1.147 & 1.858 \\
\hline
\end{tabular}

TABLE 6.2

Theoretical and measured values of $\rho\left(\mathcal{M}_{\tau}\right)$ for $(6.1)(h=1 / 16, \tau=1 / 100)$.

\begin{tabular}{ccccccc}
\hline & CN & BDF(1) & BDF(2) & BDF(3) & BDF(4) & BDF(5) \\
\hline \hline finite length & 0.050 & 0.050 & 0.052 & 0.051 & 0.049 & 0.047 \\
\hline infinite length & 0.264 & 0.069 & 0.106 & 0.170 & 0.343 & 1.184 \\
\hline measured & 0.255 & 0.064 & 0.099 & 0.161 & 0.335 & 1.166 \\
\hline
\end{tabular}

(i) if the linear multistep method is A-stable, then $\rho\left(\mathcal{M}_{\tau}\right) \leq \rho(\mathcal{M})$;

(ii) if the linear multistep method is $A(\alpha)$-stable, then

$$
\rho\left(\mathcal{M}_{\tau}\right) \leq \sup _{z \in \Sigma_{\alpha}^{c}} \rho(\mathbf{M}(z))=\sup _{z \in \partial \Sigma_{\alpha}^{c}} \rho(\mathbf{M}(z)),
$$

with $\Sigma_{\alpha}^{c}=\mathbb{C} \backslash \Sigma_{\alpha}=\{z:|\operatorname{Arg}(z)| \leq \pi-\alpha\}$.

\section{Model problem analysis.}

6.1. A one-dimensional model problem. In order to clarify the convergence behaviour of the waveform relaxation methods, we shall start with a very simple and small model problem, the one-dimensional heat equation on the unit interval,

$$
\frac{\partial \mathbf{u}}{\partial t}-\frac{\partial^{2} \mathbf{u}}{\partial x^{2}}=0, x \in[0,1] .
$$

Dirichlet boundary and initial conditions are chosen such that the solution equals $\mathbf{u}(x, t)=$ $\sin (\pi x) \exp \left(-\pi^{2} t\right)$. The problem is discretised using linear finite elements on a mesh $\Omega_{h}$ with mesh size $h=1 / 16$.

We consider the Gauss-Seidel waveform relaxation algorithm and the two-level method, with one red/black Gauss-Seidel presmoothing step, a similar postsmoothing step, standard coarsening $(H=2 h)$, and linear interpolation. The restriction is defined in the standard way for finite-element multigrid methods, i.e., $r=p^{t}$. For both waveform algorithms, we analyse the use of different time-discretisation formulae, with a constant time step $\tau=$ $1 / 100$. In particular, we consider the trapezoidal rule or Crank-Nicolson $(\mathrm{CN})$ method, and the backward differentiation formulae (BDF) of order 1 up to 5. The spectral radii of the finite and infinite time-interval operators for the standard and for the two-level algorithm are reported in Tables 6.1 and 6.2, respectively. The results were computed by direct numerical evaluation of formulae (4.11) and (4.15), and (5.7) and (5.12). The tables also present values of convergence factors, observed with an implementation of the methods, using 1000 time steps. An oscillatory initial approximation to the solution was chosen in order to excite all possible error frequencies. The measured values correspond very well to the theoretical, infinite-interval spectral radii. This effect is explained in more detail in $§ 7.1$.

These results can be understood by looking at the spectral picture [21, p. 107], which facilitates a graphical inspection of convergence. In the spectral picture a set of contour lines of the function $\rho(\mathbf{K}(z))$ or $\rho(\mathbf{M}(z))$ is plotted for $z$ in a region of the complex plane close to the complex origin. On top of this picture, the scaled stability boundary of the linear multistep methods can be plotted. Figures 6.1 and 6.2 display contour lines of $\rho(\mathbf{K}(z))$ and $\rho(\mathbf{M}(z))$ (for 


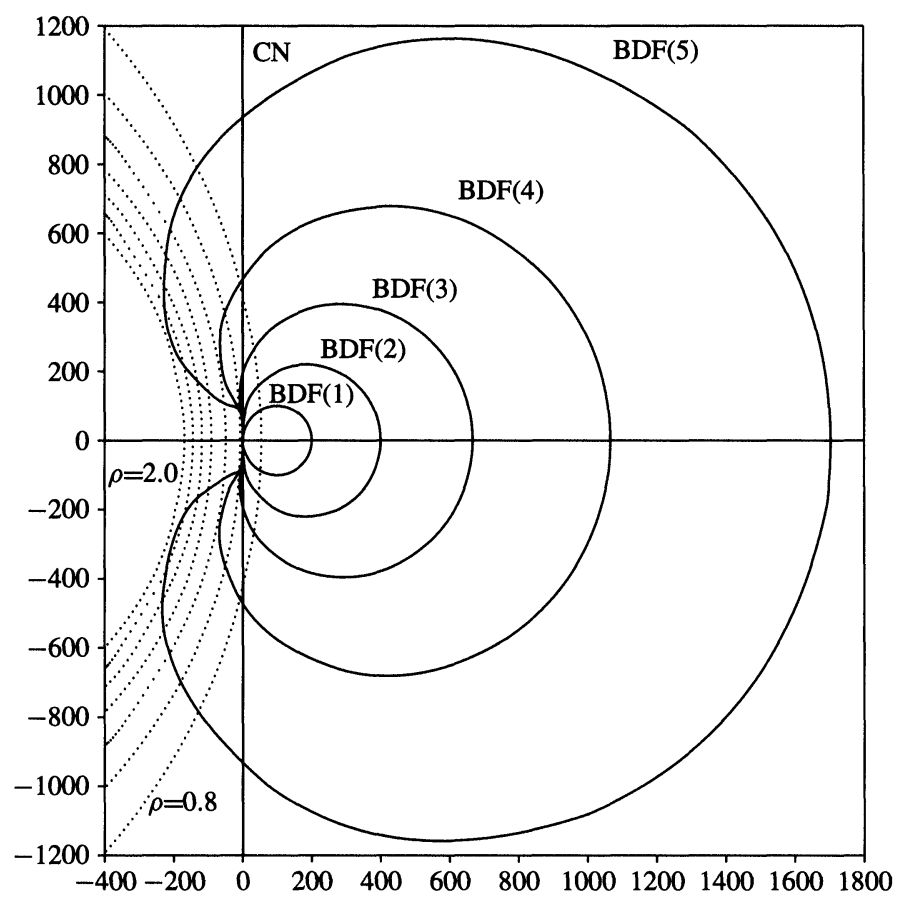

FIG. 6.1. Spectral picture and graphical convergence test for $(6.1)(\rho(K(z)), h=1 / 16, \tau=1 / 100)$.

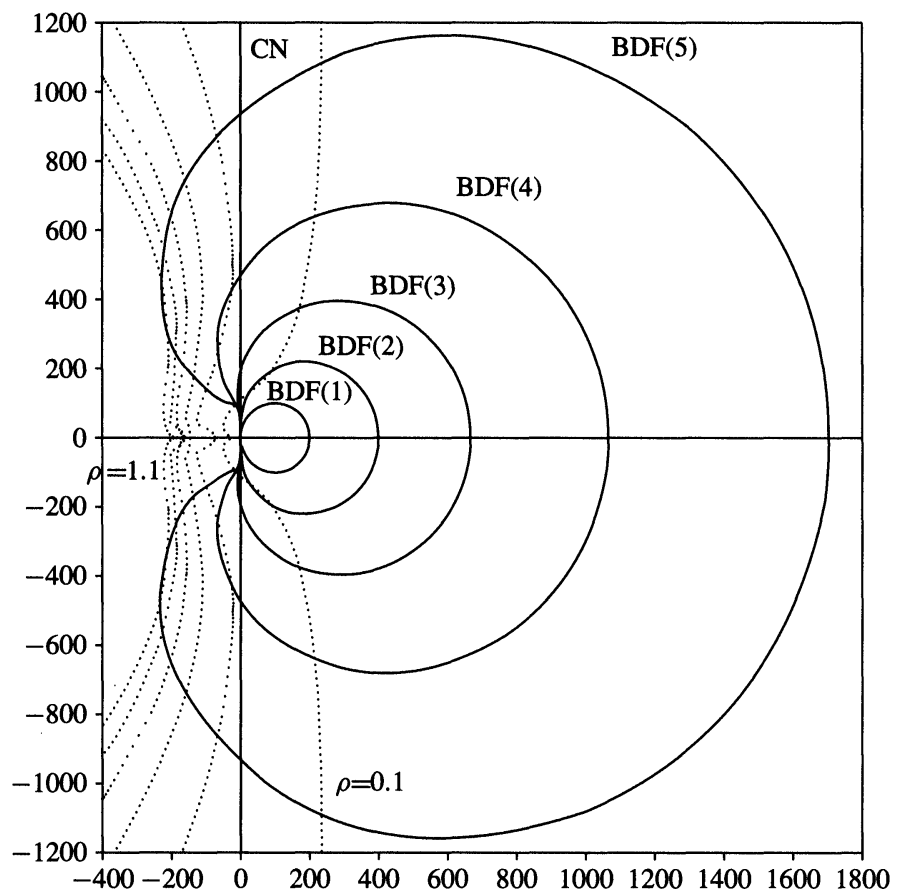

FIG. 6.2. Spectral picture and graphical convergence test for $(6.1)(\rho(\mathbf{M}(z)), h=1 / 16, \tau=1 / 100)$. 
values $0.8,1.0,1.2,1.4,1.6,1.8,2.0$ and for values $0.1,0.3,0.5,0.7,0.9,1.1$, respectively) for the model problem, together with the scaled stability region boundaries of the $\mathrm{CN}$ and BDF methods.

The values of the finite-interval spectral radii can be estimated by checking the values of the functions at the points on the real axis given by $\frac{1}{\tau} \frac{\alpha_{k}}{\beta_{k}}$ (which are not shown in the picture). With increasing order of the BDF methods, these points move to the right. Indeed, $\frac{\alpha_{k}}{\beta_{k}}$ equals $1(\mathrm{BDF}(1)), 3 / 2(\mathrm{BDF}(2)), 11 / 6(\mathrm{BDF}(3)), 25 / 12(\mathrm{BDF}(4))$, and 137/60 (BDF(5)). A value of 2 is found for the $\mathrm{CN}$ method.

The values of the infinite-interval spectral radii can be estimated by taking the maximum of $\rho(\mathbf{K}(z))$ or $\rho(\mathbf{M}(z))$ over the plotted scaled stability region boundaries. The infinite-length discrete-time waveform methods are convergent for the CN method and the low-order BDF methods. Divergence is observed for some high-order methods. In general, the spectral radius increases with increasing order of the BDF method. This was to be expected from Theorems 4.6 and 5.5, and the knowledge that the BDF methods are $A(\alpha)$-stable with $\alpha=90^{\circ}(\mathrm{BDF}(1)$, $\mathrm{BDF}(2)), \alpha=88^{\circ}(\mathrm{BDF}(3)), \alpha=73^{\circ}(\mathrm{BDF}(4))$, and $\alpha=51^{\circ}(\mathrm{BDF}(5))$. Note also that the maximum of $\rho(\mathbf{K}(z))$ over $\frac{1}{\tau} \partial S$ is found at the origin for $\mathrm{CN}, \operatorname{BDF}(1)$, and $\operatorname{BDF}(2)$. Hence, the equality of the corresponding values in Table 6.1 .

6.2. A two-dimensional model problem. Next, we study the two-dimensional heat equation on the unit square,

$$
\frac{\partial \mathbf{u}}{\partial t}-\frac{\partial^{2} \mathbf{u}}{\partial x^{2}}-\frac{\partial^{2} \mathbf{u}}{\partial y^{2}}=0, \quad(x, y) \in[0,1] \times[0,1],
$$

completed with Dirichlet boundary conditions and an initial condition. The analytical solution equals $\mathbf{u}(x, y, t)=1+\sin (\pi x / 2) \sin (\pi y / 2) \exp \left(-\pi^{2} t / 2\right)$. The problem is discretised on a regular triangular mesh with linear elements, and on a regular rectangular mesh with bilinear elements. We will analyse convergence of the two-level method, with one four-colour GaussSeidel presmoothing step, a similar postsmoothing step, standard coarsening $(H=2 h)$, and linear interpolation. The restriction is again defined by $r=p^{t}$, which leads to a seven-point formula in the linear element case, and a nine-point formula in the bilinear element case.

It is no longer practical to use a direct numerical evaluation of $\rho(\mathbf{M}(z))$ to study convergence characteristics. Instead, we can resort to Remark 5.1, which relates $\rho(\mathbf{M}(z))$ to the analysis of a standard two-grid method for a simple elliptic problem. The latter can be analysed efficiently using a classical Fourier mode analysis as introduced by Brandt in [1]. Fourier analysis shows that, under certain conditions, matrix $\mathbf{M}(z)$ is spectrally equivalent to a block-diagonal matrix whose diagonal blocks are matrices of size at most four by four. The general form of these four-by-four matrices can be derived by studying the action of the different multigrid operators on certain sets of four related exponential or sinusoidal Fourier modes. The spectral properties of $\mathbf{M}(z)$ are then calculated easily. We refer to the above reference, and to [19] and [24] for an in-depth discussion of the classical Fourier mode analysis. In the present paper we have closely followed the guidelines laid out in [24, Chap. 7].

Figure 6.3 shows the spectral picture for linear finite elements with $h=1 / 32$. In the computation we used exponential Fourier modes. They led to an exact value of the spectral radius in the case of periodic boundary conditions. A slight modification to the standard exponential mode analysis was applied to cater for the Dirichlet boundary conditions, a modification described in [24, p. 111]. Figure 6.4 shows a similar picture for bilinear elements on a grid with $h=1 / 32$. The nature of the stencil in the bilinear element case is such that a sinusoidal Fourier mode analysis is possible, see $[19, \S 7.1]$. The sinusoidal mode analysis leads automatically to the correct value of the spectral radius in the case of a problem with Dirichlet boundary conditions. 


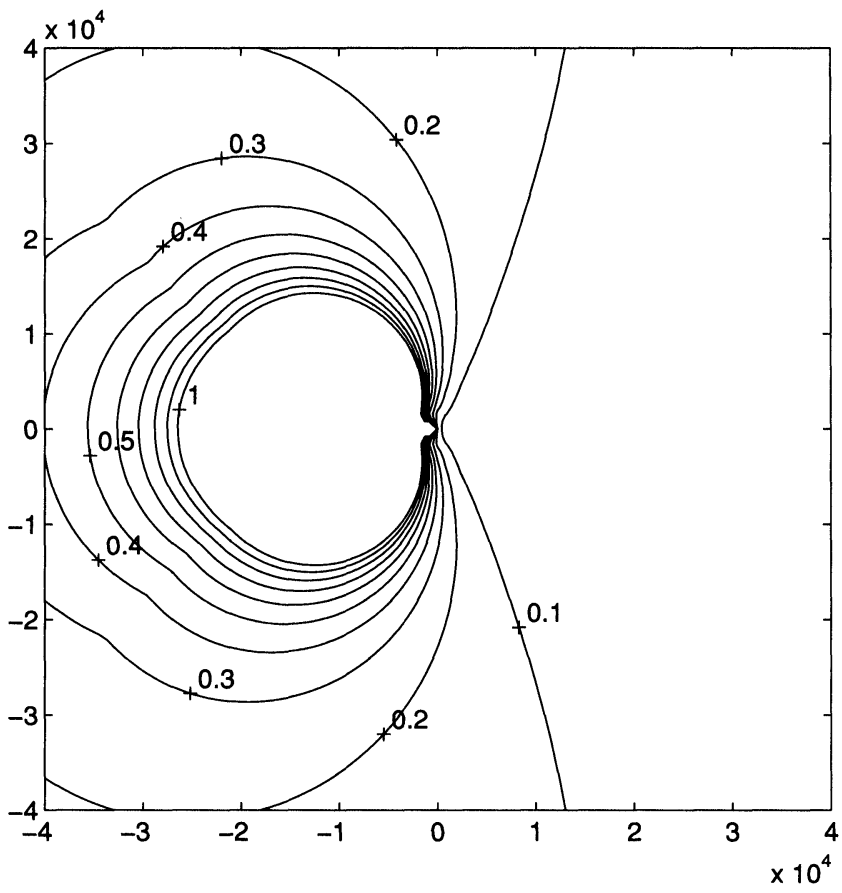

Fig. 6.3. Spectral picture for (6.2) $(\rho(\mathbf{M}(z))$, linear elements, $h=1 / 32)$.

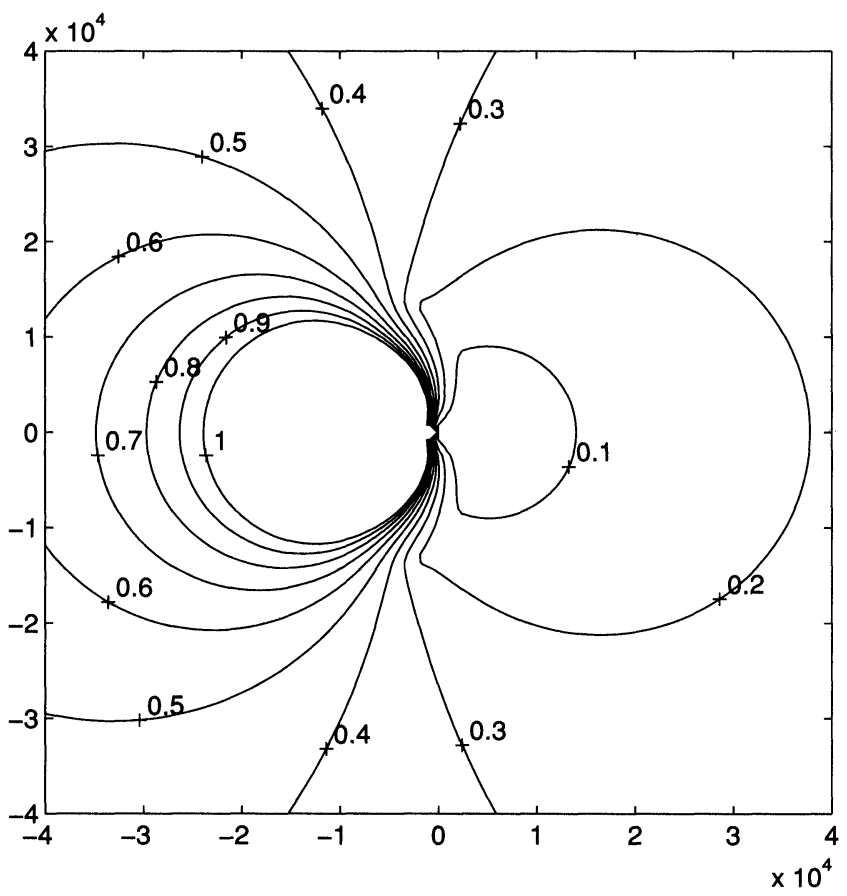

Fig. 6.4. Spectral picture for (6.2) $(\rho(\mathbf{M}(z))$, bilinear elements, $h=1 / 32)$. 
TABLE 6.3

Theoretical and measured values of $\rho\left(\mathcal{M}_{\tau}\right)$ for (6.2) (linear elements, $h=1 / 32, \tau=1 / 100$ ).

\begin{tabular}{ccccccc}
\hline & $\mathrm{CN}$ & $\mathrm{BDF}(1)$ & $\mathrm{BDF}(2)$ & $\mathrm{BDF}(3)$ & $\mathrm{BDF}(4)$ & $\mathrm{BDF}(5)$ \\
\hline \hline finite length & 0.102 & 0.120 & 0.110 & 0.104 & 0.100 & 0.097 \\
\hline infinite length & 0.374 & 0.148 & 0.148 & 0.150 & 0.170 & 0.233 \\
\hline measured & 0.329 & 0.135 & 0.137 & 0.138 & 0.150 & 0.198 \\
\hline
\end{tabular}

TABLE 6.4

Theoretical and measured values of $\rho\left(\mathcal{M}_{\tau}\right)$ for (6.2) (bilinear elements, $h=1 / 32, \tau=1 / 100$ ).

\begin{tabular}{ccccccc}
\hline & $\mathrm{CN}$ & $\mathrm{BDF}(1)$ & $\mathrm{BDF}(2)$ & $\mathrm{BDF}(3)$ & $\mathrm{BDF}(4)$ & $\mathrm{BDF}(5)$ \\
\hline \hline finite length & 0.038 & 0.042 & 0.040 & 0.038 & 0.037 & 0.036 \\
\hline infinite length & 0.356 & 0.052 & 0.058 & 0.068 & 0.087 & 0.132 \\
\hline measured & 0.313 & 0.039 & 0.044 & 0.049 & 0.067 & 0.118 \\
\hline
\end{tabular}

In Tables 6.3 and 6.4 we present two-grid spectral radii for the finite-length and infinitelength waveform operators, together with two-grid convergence factors computed numerically using an oscillatory initial approximation and 1000 time steps. As for the one-dimensional problem, there is again a good agreement between the theoretical infinite-length spectral radii and the experimental convergence factors.

\section{Numerical experiments.}

7.1. The one-dimensional model problem. In this section, we shall clarify the relation between the finite and infinite time-interval spectral radii. To this end, we solve (6.1) using the Gauss-Seidel waveform method with $\mathrm{BDF}(2)$ and $\mathrm{BDF}(5)$ time discretisation, with constant time step $\tau=1 / 100$ on 100 time levels $(N=100)$. (Note that a similar analysis could be done for the two-level method and/or for larger values of $N$. It would lead to similar conclusions and insights.)

Let $d_{\tau}^{(v)}$ denote the discrete defect or residual in the $\nu$ th iteration. The convergence factor of the $v$ th iteration is then defined by

$$
\rho^{(\nu)}=\left\|d_{\tau}^{(v)}\right\|_{2} /\left\|d_{\tau}^{(\nu-1)}\right\|_{2} .
$$

In Fig. 7.1 successive convergence factors are plotted for the first 400 waveform Gauss-Seidel iterations, when $\mathrm{BDF}(2)$ discretisation is used. These factors appear to remain more or less constant for a large number of iterations. The height of the plateau matches the value obtained in Table 6.1 for infinite time intervals, i.e., 0.962. Eventually, the plateau in Fig. 7.1 is left, and the factors start to decrease. Ultimately, they start to rise again and reach the value 1 . This is for purely technical reasons, because at that time the solution has converged within the finite-precision arithmetic of the implementation. A similar plot is given in Fig. 7.2 for the $\mathrm{BDF}(5)$ discretisation. Here, the evolution is much more erratic. The results clearly indicate divergence for a large number of iterations. After a sufficient number of iterations, the convergence factors decrease below 1 , and the iteration starts to converge rapidly.

This behaviour can be explained by examining the time-level convergence factors. These factors are similar to the standard convergence factors (7.1), but are evaluated for each time level separately,

$$
\rho_{k}^{(\nu)}=\left\|d_{k}^{(\nu)}\right\|_{2} /\left\|d_{k}^{(\nu-1)}\right\|_{2} .
$$

In Fig. 7.3, we plotted such time-level convergence factors for the $\operatorname{BDF}(2)$ method (for $v=10, v=100, v=200$, and $v=300$ ). The factor measured at the first time level equals 


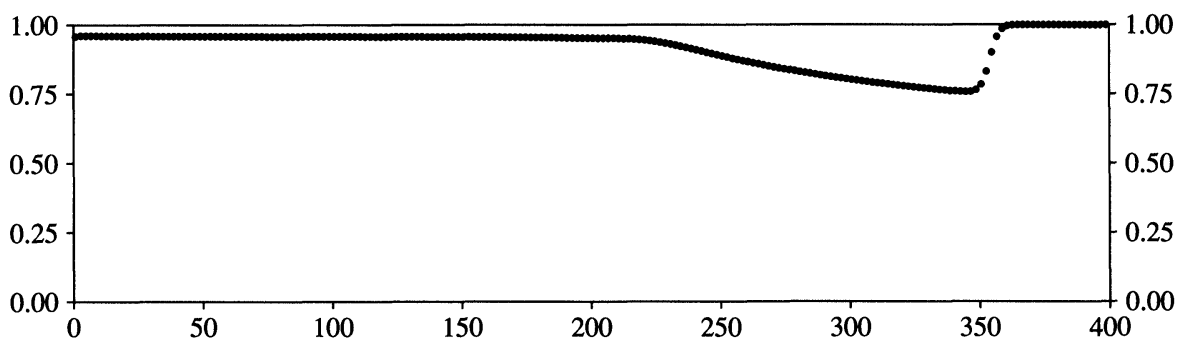

Fig. 7.1. Convergence factors $\rho^{(v)}$ as a function of $v(B D F(2)$ method $)$.

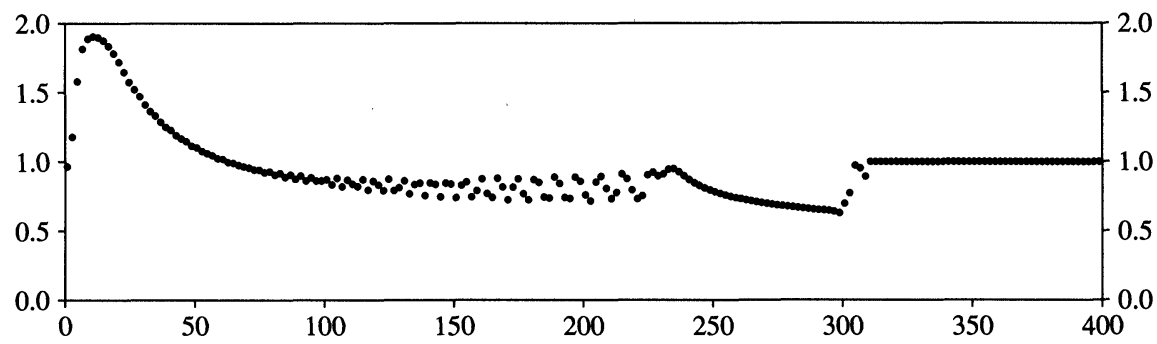

FIG. 7.2. Convergence factors $\rho^{(v)}$ as a function of $v(B D F(5)$ method) .
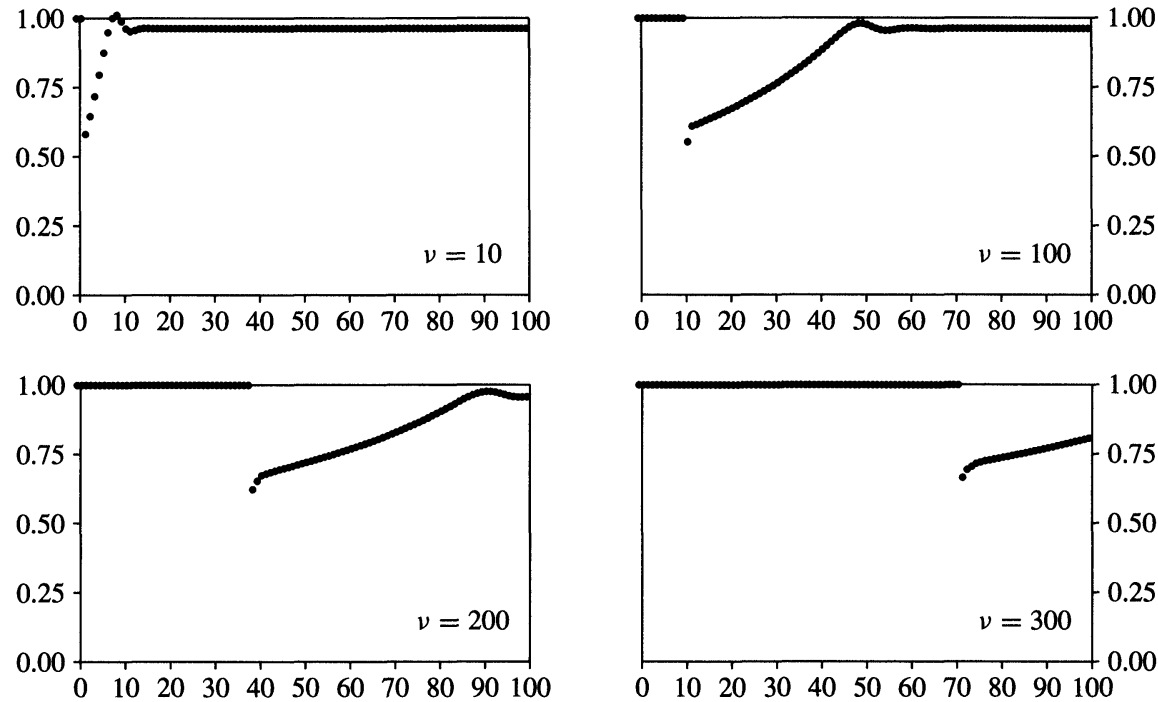

Fig. 7.3. Time-level convergence factors $\rho_{k}^{(v)}$ as a function of $k(B D F(2)$ method).

0.548 , exactly equal to the value predicted by the finite time-interval analysis in Table 6.1. The convergence factors at the next time levels increase, and eventually become constant. The height of the plateau matches the spectral radius value for infinite time intervals. As more iterations are applied, the plateau is forced out of the time window and the corresponding convergence factors decrease.

In Fig. 7.4, we have plotted time-level convergence factors for the $\operatorname{BDF}(5)$ method (for $v=1, v=5, v=50$, and $v=100$ ). Again, we observe that the factor at the first time level corresponds to the value predicted by the finite time-interval analysis $(0.414)$. The pictures illustrate the onset of oscillations which rapidly explode. As more iterations are applied, the 

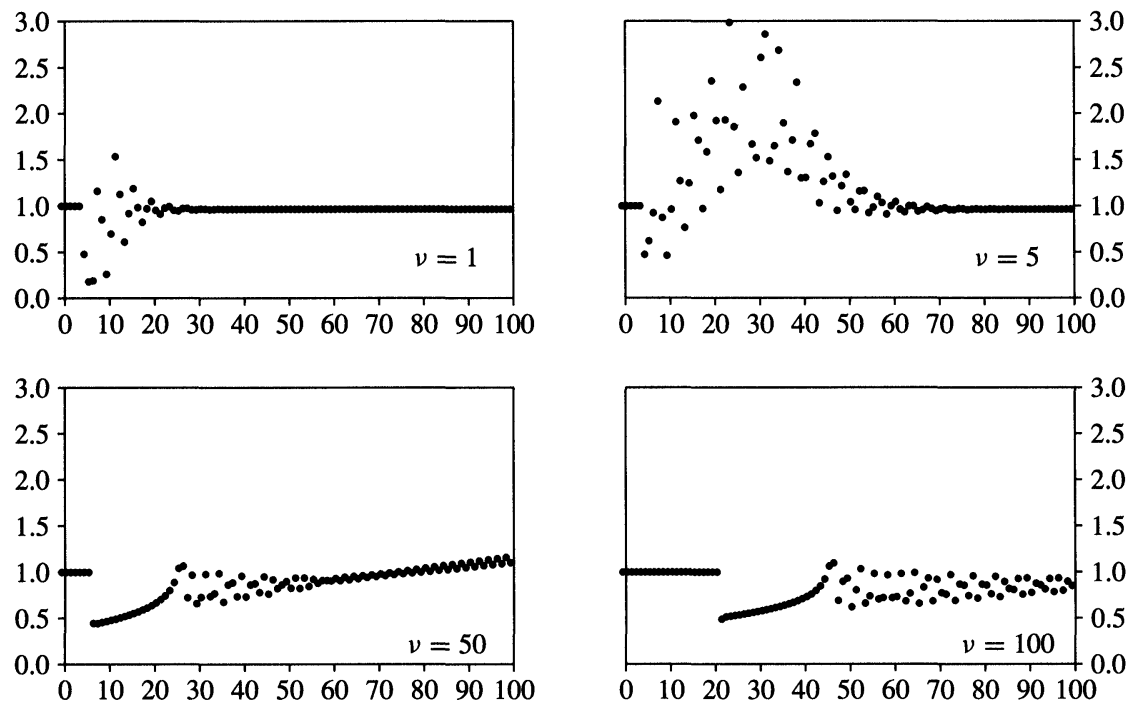

FIG. 7.4. Time-level convergence factors $\rho_{k}^{(v)}$ as a function of $k(B D F(5)$ method $)$.

TABLE 7.1

Averaged convergence factors for (6.2) (linear basis functions, $h=1 / 32, \tau=1 / 200$ ).

\begin{tabular}{ccccccc}
\hline & CN & BDF(1) & BDF(2) & BDF(3) & BDF(4) & BDF(5) \\
\hline \hline Gauss-Seidel & 0.990 & 0.990 & 0.990 & 0.997 & - & - \\
\hline$V$-cycle & 0.438 & 0.177 & 0.177 & 0.275 & 0.844 & - \\
\hline$W$-cycle & 0.307 & 0.124 & 0.124 & 0.124 & 0.381 & - \\
\hline
\end{tabular}

TABLE 7.2

Averaged convergence factors for (6.2) (bilinear basis functions, $h=1 / 32, \tau=1 / 200$ ).

\begin{tabular}{ccccccc}
\hline & CN & BDF(1) & BDF(2) & BDF(3) & BDF(4) & BDF(5) \\
\hline \hline Gauss-Seidel & 0.985 & 0.985 & 0.985 & 0.996 & - & - \\
\hline$V$-cycle & 0.446 & 0.046 & 0.132 & 0.332 & - & - \\
\hline$W$-cycle & 0.295 & 0.041 & 0.041 & 0.052 & 0.538 & - \\
\hline
\end{tabular}

region of divergent behaviour moves to the right, and is forced out of the time window. From then on, the iteration converges rapidly.

7.2. The two-dimensional problem. We discretise (6.2) using linear basis functions (triangular elements) or bilinear basis functions (rectangular elements) on a mesh with equal mesh size in $x$ - and $y$-direction. The resulting system of ODEs is solved using Gauss-Seidel and multigrid waveform relaxation for $t \in[0,1]$. In the latter we applied $V$-cycles and $W$ cycles, with one presmoothing and one postsmoothing step of four-colour Gauss-Seidel type. We use standard coarsening down to a mesh with size $h=1 / 2$, seven-point prolongation (linear basis functions), and nine-point prolongation (bilinear basis functions). The restriction operator is defined as $r=p^{t}$.

In Tables 7.1 and 7.2 we report averaged convergence factors. These are defined as the average of $\rho^{(v)}$ over the region of nearly constant behaviour. The dashes ("-") in the tables indicate that the corresponding method showed divergence over a large number of iterations. Both tables illustrate the dependence of the convergence on the nature of the time-discretisation method. 
In Tables 7.3-7.8, we report averaged convergence factors obtained with $W$-cycles for different values of the mesh-size parameters, and for different discretisation schemes. We observe a dependence of the actual convergence factors on $h$ and $\tau$. For the Crank-Nicolson and $\mathrm{BDF}(2)$ methods, these factors appear to be bounded by a constant, smaller than one, independent of the mesh size.

For a constant value of $h$, we expect the convergence factors to converge to the continuoustime results when $\tau$ decreases, see $\S \S 4.3$ and 5.3. This behaviour is recognised clearly for the CN method in Tables 7.3 and 7.6. Due to the shape of the stability regions of the BDF(2) and BDF(4) methods, it takes a much smaller value of $\tau$ before the discrete-time convergence factors tend to the continuous-time ones, see Tables 7.4, 7.5, 7.7, and 7.8. For a constant value of $\tau$, we observe an initial increase of the convergence factor when $h$ decreases. For sufficiently small $h$ the convergence factor starts to decrease again. This behaviour is similar to what is observed when the multigrid waveform relaxation method is used to solve the ODEs obtained by spatial finite-difference discretisation of a parabolic problem. We refer to [21, $\S 3.5]$ for an intuitive explanation, and to [22] for a discussion based on an exponential Fourier mode analysis.

Appendix. Analysis of the multigrid waveform relaxation operators. We consider the case where we have a hierarchy of grids, $\Omega_{h_{0}} \subset \Omega_{h_{1}} \subset \cdots \subset \Omega_{h_{l}}$, a set of prolongation operators $p_{h_{i}}^{h_{i+1}}: \Omega_{h_{i}} \rightarrow \Omega_{h_{i+1}}, 0 \leq i \leq l-1$, a set of restriction operators, $r_{h_{i+1}}^{h_{i}}$ : $\Omega_{h_{i+1}} \longrightarrow \Omega_{h_{i}}, 0 \leq i \leq l-1$, and discretisation matrices $B_{h_{i}}$ and $A_{h_{i}}, 0 \leq i \leq l$. The multigrid algorithm differs from the two-grid cycle in that the coarse-grid defect equation is approximately solved by an application of $\gamma$ two-grid cycles, an idea that is further extended recursively. (The classical $V$ - and $W$-cycles correspond to $\gamma=1$ and $\gamma=2$, respectively.) In the continuous-time case this leads to an iteration of the form $u^{(v)}=\mathcal{M}_{h_{l}} u^{(v-1)}+\varphi$. In the discrete-time case we end up with an iteration operator which we denote by $\left(\mathcal{M}_{h_{l}}\right)_{\tau}$.

Both iterative schemes can be analysed in exactly the same way as the two-grid cycles have been analysed. A Laplace-transform argument is used in the continuous-time case, whereas the discrete-time case is treated by using a discrete Laplace-transform method. Proceeding as before, we can derive the symbol of the continuous-time multigrid waveform relaxation method, $\mathbf{M}_{\mathbf{h}_{1}}(z)$. The latter takes a particularly simple form under the natural assumption that the semidiscretised PDE operators are invertible. In that case we can apply the following Lemma.

LEMMA A.1. Let $B \dot{u}+A u=f$ have a unique solution, and let it be solved approximately by $\gamma$ steps of a consistent waveform method: $u^{(k)}=\mathcal{M} u^{(k-1)}+\varphi$ with $u^{(0)}(t)=0$. Then, the $\gamma$ th iterate can be represented as $u^{(\gamma)}=\left(I-\mathcal{M}^{\gamma}\right) u$.

Under the above assumption, the multigrid symbol becomes

$$
\mathbf{M}_{\mathbf{h}_{1}}(z)= \begin{cases}\mathbf{K}_{\mathbf{h}_{1}}^{\nu_{2}}(z)\left(I-p_{h_{l-1}}^{h_{l}}\left(I-\mathbf{M}_{\mathbf{h}_{1-1}}^{\gamma}(z)\right) \mathbf{L}_{\mathbf{h}_{1-1}}^{-1}(z) r_{h_{l}}^{h_{l-1}} \mathbf{L}_{\mathbf{h}_{1}}(z)\right) \mathbf{K}_{\mathbf{h}_{\mathbf{1}}}^{\nu_{1}}(z), & l \neq 1, \\ \mathbf{K}_{\mathbf{h}_{\mathbf{1}}}^{\nu_{2}}(z)\left(I-p_{h_{0}}^{h_{1}} \mathbf{L}_{\mathbf{h}_{0}}^{-1}(z) r_{h_{1}}^{h_{0}} \mathbf{L}_{\mathbf{h}_{1}}(z)\right) \mathbf{K}_{\mathbf{h}_{1}}^{\nu_{1}}(z), & l=1,\end{cases}
$$

where $\mathbf{L}_{\mathbf{h}_{\mathbf{i}}}(z)=z B_{h_{i}}+A_{h_{i}}$ and $\mathbf{K}_{\mathbf{h}_{\mathbf{i}}}(z)=\left(z M_{B_{h_{i}}}+M_{A_{h_{i}}}\right)^{-1}\left(z N_{B_{h_{i}}}+N_{A_{h_{i}}}\right)$. Note that $\mathbf{M}_{\mathbf{h}_{\mathbf{l}}}(z)$ is technically more complicated when the assumption is violated. In that case, it does not involve the factor $\mathbf{L}_{\mathbf{h}_{1-1}}^{-1}(z)$.

Remark A.1. Let $\mathbf{K}_{\mathbf{h}_{\mathbf{i}}}(z)$ correspond to a Gauss-Seidel or weighted Jacobi splitting. Then, $\mathbf{M}_{\mathbf{h}_{1}}(z)$ is the multigrid iteration operator for the elliptic problem $\left(z B_{h_{l}}+A_{h_{l}}\right) u_{h_{l}}=f_{h_{l}}$; compare $[4$, p. 162] and $[19$, p. 46]. 
TABLE 7.3

Averaged convergence factors for (6.2) (linear basis functions, CN method, W-cycle).

\begin{tabular}{c||cccccc}
\hline$h, \tau$ & 0.04 & 0.02 & 0.01 & 0.005 & 0.0025 & 0.001 \\
\hline \hline $1 / 4$ & 0.103 & 0.135 & 0.134 & 0.135 & 0.134 & 0.135 \\
\hline $1 / 8$ & 0.126 & 0.256 & 0.305 & 0.304 & 0.304 & 0.304 \\
\hline $1 / 16$ & 0.117 & 0.135 & 0.282 & 0.359 & 0.358 & 0.357 \\
\hline $1 / 32$ & 0.123 & 0.125 & 0.140 & 0.307 & 0.372 & 0.371 \\
\hline
\end{tabular}

TABLE 7.4

Averaged convergence factors for (6.2) (linear basis functions, $B D F(2)$ method, $W$-cycle).

\begin{tabular}{c||cccccc}
\hline$h, \tau$ & 0.04 & 0.02 & 0.01 & 0.005 & 0.0025 & 0.001 \\
\hline \hline $1 / 4$ & 0.051 & 0.070 & 0.111 & 0.128 & 0.133 & 0.134 \\
\hline $1 / 8$ & 0.086 & 0.086 & 0.108 & 0.194 & 0.247 & 0.291 \\
\hline $1 / 16$ & 0.118 & 0.118 & 0.118 & 0.118 & 0.124 & 0.266 \\
\hline $1 / 32$ & 0.124 & 0.124 & 0.124 & 0.124 & 0.124 & 0.125 \\
\hline
\end{tabular}

TABLE 7.5

Averaged convergence factors for (6.2) (linear basis functions, BDF(4) method, W-cycle).

\begin{tabular}{c||cccccc}
\hline$h, \tau$ & 0.04 & 0.02 & 0.01 & 0.005 & 0.0025 & 0.001 \\
\hline \hline $1 / 4$ & 0.173 & 0.320 & 0.290 & 0.171 & 0.135 & 0.134 \\
\hline $1 / 8$ & 0.141 & 0.324 & 0.525 & 0.766 & 0.666 & 0.311 \\
\hline $1 / 16$ & 0.121 & 0.154 & 0.358 & 0.653 & 0.807 & 0.948 \\
\hline $1 / 32$ & 0.124 & 0.124 & 0.124 & 0.381 & 0.726 & 1.091 \\
\hline
\end{tabular}

TABLE 7.6

Averaged convergence factors for (6.2) (bilinear basis functions, $C N$ method, $W$-cycle).

\begin{tabular}{c||cccccc}
\hline$h, \tau$ & 0.04 & 0.02 & 0.01 & 0.005 & 0.0025 & 0.001 \\
\hline \hline $1 / 4$ & 0.102 & 0.133 & 0.136 & 0.137 & 0.137 & 0.137 \\
\hline $1 / 8$ & 0.150 & 0.231 & 0.285 & 0.293 & 0.294 & 0.294 \\
\hline $1 / 16$ & 0.080 & 0.179 & 0.268 & 0.330 & 0.343 & 0.344 \\
\hline $1 / 32$ & 0.042 & 0.086 & 0.184 & 0.295 & 0.343 & 0.355 \\
\hline
\end{tabular}

TABLE 7.7

Averaged convergence factors for (6.2) (bilinear basis functions, $B D F(2)$ method, $W$-cycle).

\begin{tabular}{c||cccccc}
\hline$h, \tau$ & 0.04 & 0.02 & 0.01 & 0.005 & 0.0025 & 0.001 \\
\hline \hline $1 / 4$ & 0.049 & 0.072 & 0.085 & 0.088 & 0.106 & 0.125 \\
\hline $1 / 8$ & 0.063 & 0.088 & 0.130 & 0.178 & 0.224 & 0.241 \\
\hline $1 / 16$ & 0.045 & 0.046 & 0.047 & 0.104 & 0.167 & 0.246 \\
\hline $1 / 32$ & 0.041 & 0.041 & 0.041 & 0.041 & 0.042 & 0.132 \\
\hline
\end{tabular}

TABLE 7.8

Averaged convergence factors for (6.2) (bilinear basis functions, BDF(4) method, $W$-cycle).

\begin{tabular}{c||cccccc}
\hline$h, \tau$ & 0.04 & 0.02 & 0.01 & 0.005 & 0.0025 & 0.001 \\
\hline $1 / 4$ & 0.124 & 0.217 & 0.161 & 0.148 & 0.139 & 0.135 \\
\hline $1 / 8$ & 0.158 & 0.319 & 0.600 & 0.661 & 0.405 & 0.324 \\
\hline $1 / 16$ & 0.069 & 0.147 & 0.377 & 0.735 & 0.892 & 0.770 \\
\hline $1 / 32$ & 0.042 & 0.048 & 0.112 & 0.538 & 0.646 & 0.937 \\
\hline
\end{tabular}


As in [10], the continuous-time convergence theorems can be formulated in terms of this symbol. The ideas behind the proofs are identical to the ones behind the corresponding proofs in the above reference, and therefore omitted.

THEOREM A.2. The multigrid waveform relaxation operator $\mathcal{M}_{h_{l}}$ is a bounded operator in $C[0, T]$ and

$$
\rho\left(\mathcal{M}_{h_{l}}\right)=\rho\left(\mathbf{M}_{\mathbf{h}_{1}}(\infty)\right)
$$

THEOREM A.3. Assume that all eigenvalues of $M_{B_{h_{i}}}^{-1} M_{A_{h_{i}}}, 1 \leq i \leq l$, and $B_{h_{0}}^{-1} A_{h_{0}}$ have positive real parts, and consider $\mathcal{M}_{h_{l}}$ as an operator in $L_{p}(0, \infty)$ with $1 \leq p \leq \infty$. Then, $\mathcal{M}_{h_{l}}$ is a bounded operator with spectral radius

$$
\rho\left(\mathcal{M}_{h_{l}}\right)=\sup _{\operatorname{Re}(z) \geq 0} \rho\left(\mathbf{M}_{\mathbf{h}_{1}}(z)\right)=\sup _{\xi \in \mathbb{R}} \rho\left(\mathbf{M}_{\mathbf{h}_{1}}(i \xi)\right) .
$$

Following the line of arguments of $\S 5.1$, we can derive the discrete-time symbol

$$
\left(\mathbf{M}_{\mathrm{h}_{1}}\right)_{\tau}(z)=\mathbf{M}_{\mathbf{h}_{1}}\left(\frac{1}{\tau} \frac{a}{b}(z)\right) .
$$

The discrete-time convergence theorems are immediate extensions of Theorems 5.1 and 5.3. The proofs are very similar.

THEOREM A.4. Assume $\frac{\alpha_{k}}{\beta_{k}} \notin \bigcup_{i=1}^{l} \sigma\left(-\tau M_{B_{h_{i}}}^{-1} M_{A_{h_{i}}}\right) \cup \sigma\left(-\tau B_{h_{0}}^{-1} A_{h_{0}}\right)$, and consider $\left(\mathcal{M}_{h_{l}}\right)_{\tau}$ as an operator in $l_{p}(N)$, with $1 \leq p \leq \infty$ and $N$ finite. Then, $\left(\mathcal{M}_{h_{l}}\right)_{\tau}$ is a bounded operator and

$$
\rho\left(\left(\mathcal{M}_{h_{l}}\right)_{\tau}\right)=\rho\left(\mathbf{M}_{\mathbf{h}_{1}}\left(\frac{1}{\tau} \frac{\alpha_{k}}{\beta_{k}}\right)\right)
$$

THEOREM A.5. Assume $\bigcup_{i=1}^{l} \sigma\left(-\tau M_{B_{h_{i}}}^{-1} M_{A_{h_{i}}}\right) \cup \sigma\left(-\tau B_{h_{0}}^{-1} A_{h_{0}}\right) \subset$ int $S$, and consider $\left(\mathcal{M}_{h_{l}}\right)_{\tau}$ as an operator in $l_{p}(\infty)$, with $1 \leq p \leq \infty$. Then,

$$
\rho\left(\left(\mathcal{M}_{h_{l}}\right)_{\tau}\right)=\sup \left\{\rho\left(\mathbf{M}_{\mathbf{h}_{1}}(z)\right) \mid \tau z \in \mathbb{C} \backslash \text { int } S\right\}=\sup _{\tau z \in \partial S} \rho\left(\mathbf{M}_{\mathbf{h}_{1}}(z)\right)
$$

\section{REFERENCES}

[1] A. BRANDT, Multi-level adaptive solutions to boundary-value problems, Math. Comp., 31 (1977), pp. 333-390.

[2] P. BRENNER, V. Thomé, AND L. B. WAHLBIN, Besov Spaces and Applications to Difference Methods for Initial Value Problems, Lecture Notes in Mathematics 434, Springer-Verlag, Berlin, 1975.

[3] W. HackBusCh, Parabolic multi-grid methods, in Computing Methods in Applied Sciences and Engineering VI, R. Glowinski and J.-L. Lions, eds., North-Holland, Amsterdam, 1984, pp. 189-197.

[4] - Multi-grid methods and applications, Springer Series in Computational Mathematics 4, SpringerVerlag, Berlin, 1985.

[5] E. HAIRER AND G. WANNER, Solving ordinary differential equations II, Springer Series in Computational Mathematics 14, Springer-Verlag, Berlin, 1991.

[6] G. H. HaRdy, J. E. Litrlewood, AND G. Pólya, Inequalities, 2nd ed., Cambridge University Press, Cambridge, 1978.

[7] G. Horton, The time-parallel multigrid method, Comm. Appl. Numer. Methods, 8 (1992), pp. 585-595.

[8] G. HoRTON AND S. VANDEWALlE, A space-time multigrid method for parabolic Partial Differential Equations, SIAM J. Sci. Comput., 16 (1995), pp. 848-864.

[9] G. HORTON, S. VANDEWALLE, AND P. WORLEY, An algorithm with polylog parallel complexity for solving parabolic partial differential equations, SIAM J. Sci. Comput., 16 (1995), pp. 531-541. 
[10] J. JANSSEN AND S. VANDEWALLE, Multigrid waveform relaxation on spatial finite element meshes: The continuous-time case, SIAM J. Numer. Anal., 33 (1996).

[11] J. D. LAMBERT, Computational Methods in Ordinary Differential Equations, John Wiley and Sons, Chichester, 1973.

[12] E. Lelarasmee, A. E. Ruehli, AND A. L. Sangiovanni-Vincentelli, The waveform relaxation method for time-domain analysis of large scale integrated circuits, IEEE Trans. CAD, 1 (1982), pp. 131-145.

[13] C. LUBICH, On the stability of linear multistep methods for Volterra convolution equations, IMA J. Numer. Anal., 3 (1983), pp. 439-465.

[14] C. LUBich AND A. OstermanN, Multi-grid dynamic iteration for parabolic equations, BIT, 27 (1987), pp. 216-234.

[15] U. MiekKala AND O. NevanlinNa, Convergence of dynamic iteration methods for initial value problems, SIAM J. Sci. Statist. Comput., 8 (1987), pp. 459-482.

[16] - Sets of convergence and stability regions, BIT, 27 (1987), pp. 554-584.

[17] O. NevanlinNa, Remarks on Picard-Lindelöf iteration, II, BIT, 29 (1989), pp. 535-562.

[18] L. ReICHEL AND L. N. TREFETHEN, Eigenvalues and pseudo-eigenvalues of Toeplitz matrices, Linear Algebra Appl., 162-164 (1992), pp. 153-185.

[19] K. STÜBEN AND U. TROTTENBERG, Multigrid methods: Fundamental algorithms, model problem analysis and applications, in Multigrid Methods, U. Trottenberg and W. Hackbusch, eds., Lecture Notes in Mathematics 960, Springer-Verlag, Berlin, 1982, pp. 1-176.

[20] V. THOмÉ, Galerkin finite element methods for parabolic problems, Lecture Notes in Mathematics 1054, Springer-Verlag, Berlin, 1984.

[21] S. VANDEWalle, Parallel Multigrid Waveform Relaxation for Parabolic Problems, Teubner, Stuttgart, 1993.

[22] S. VANDEWALlE AND G. HoRTON, Fourier mode analysis of the multigrid waveform relaxation and time-parallel multigrid methods, Computing, 54(4), (1995), pp. 317-330.

[23] S. VANDEWAlle AND E. VAN DE Velde, Space-time concurrent multigrid waveform relaxation, Annals of Numerical Mathematics, 1 (1994), pp. 347-363.

[24] P. Wesseling, An Introduction to Multigrid Methods, John Wiley and Sons, Chichester, 1992.

[25] K. Yosida, Functional Analysis, Springer-Verlag, New York, 1980. 